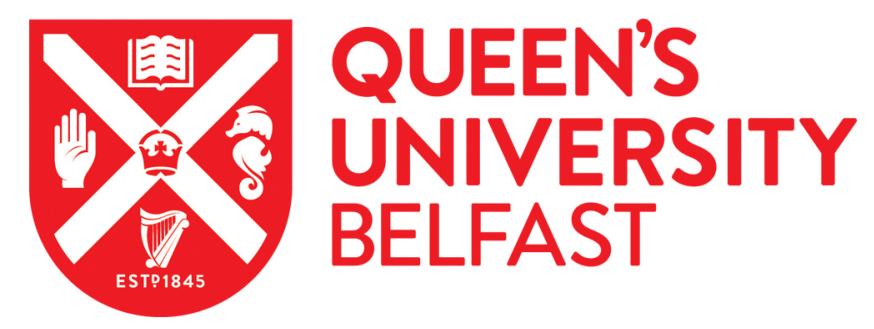

\title{
Molecular Hydrogen Emission from Protoplanetary Disks: Effects of X- ray Irradiation and Dust Evolution
}

Nomura, H., Aikawa, Y., Tsujimoto, M., Nakagawa, Y., \& Millar, TJ. (2007). Molecular Hydrogen Emission from Protoplanetary Disks: Effects of X-ray Irradiation and Dust Evolution. Astrophysical Journal, 661(1 I), 334-353. https://doi.org/10.1086/513419

Published in:

Astrophysical Journal

Queen's University Belfast - Research Portal:

Link to publication record in Queen's University Belfast Research Portal

\section{General rights}

Copyright for the publications made accessible via the Queen's University Belfast Research Portal is retained by the author(s) and / or other copyright owners and it is a condition of accessing these publications that users recognise and abide by the legal requirements associated with these rights.

Take down policy

The Research Portal is Queen's institutional repository that provides access to Queen's research output. Every effort has been made to ensure that content in the Research Portal does not infringe any person's rights, or applicable UK laws. If you discover content in the Research Portal that you believe breaches copyright or violates any law, please contact openaccess@qub.ac.uk. 
The Astrophysical Journal, 661:334-353, 2007 May 20

(C) 2007. The American Astronomical Society. All rights reserved. Printed in U.S.A.

\title{
MOLECULAR HYDROGEN EMISSION FROM PROTOPLANETARY DISKS. II. EFFECTS OF X-RAY IRRADIATION AND DUST EVOLUTION
}

\author{
H. Nomura, ${ }^{1,2}$ Y. Aikawa, ${ }^{1}$ M. Tsujimoto, ${ }^{3}$ Y. Nakagawa, ${ }^{1}$ and T. J. Millar ${ }^{2}$ \\ Received 2006 December 5; accepted 2007 January 31
}

\begin{abstract}
Detailed models for the density and temperature profiles of gas and dust in protoplanetary disks are constructed by taking into account X-ray and UV irradiation from a central T Tauri star, as well as dust size growth and settling toward the disk midplane. The spatial and size distributions of dust grains are numerically computed by solving the coagulation equation for settling dust particles, with the result that the mass and total surface area of dust grains per unit volume of the gas in the disks are very small, except at the midplane. The $\mathrm{H}_{2}$ level populations and line emission are calculated using the derived physical structure of the disks. X-ray irradiation is the dominant heating source of the gas in the inner disk and in the surface layer, while the UV heating dominates otherwise. If the central star has strong X-ray and weak UV radiation, the $\mathrm{H}_{2}$ level populations are controlled by X-ray pumping, and the X-ray-induced transition lines could be observable. If the UV irradiation is strong, the level populations are controlled by thermal collisions or UV pumping, depending on the dust properties. As the dust particles evolve in the disks, the gas temperature at the disk surface drops because the grain photoelectric heating becomes less efficient. This makes the level populations change from LTE to non-LTE distributions, which results in changes to the line ratios. Our results suggest that dust evolution in protoplanetary disks could be observable through the $\mathrm{H}_{2}$ line ratios. The emission lines are strong from disks irradiated by strong UV and X-rays and possessing small dust grains; such disks will be good targets in which to observe $\mathrm{H}_{2}$ emission.
\end{abstract}

Subject headings: line: formation — molecular processes — planetary systems: protoplanetary disks radiative transfer

\section{INTRODUCTION}

Observations of thermal dust continuum emission (e.g., Kenyon \& Hartmann 1987; Beckwith \& Sargent 1993; Kitamura et al. 2002) and, more directly, images of light scattered from dust grains (e.g., Roddier et al. 1996; Itoh et al. 2002; Duchene et al. 2004) have revealed that young stellar objects have circumstellar disks. In addition, a gaseous component has been detected from the circumstellar disks around T Tauri stars (e.g., Carr 1989; Dutrey et al. 1997, 2007; Thi et al. 2004; Qi et al. 2006; Najita et al. 2007; Bergin et al. 2007). Recent high spectral resolution and highsensitivity observations have made it possible to detect line emission of molecular hydrogen gas, which is the major component of the gas in the protoplanetary disks (Thi et al. 1999, 2001a, 2001b; Richter et al. 2002; Sheret et al. 2003; Sako et al. 2005; Weintraub et al. 2000; Bary et al. 2002, 2003; Itoh et al. 2003; Herczeg et al. 2002, 2004, 2006; Bergin et al. 2004). Furthermore, detection of molecular hydrogen lines in the near-infrared (NIR) and midinfrared (MIR) wavelength bands has been reported toward T Tauri stars and Herbig Ae/Be stars very recently (Weintraub et al. 2005; J. S. Bary et al. 2007, in preparation; Richter et al. 2007; Bitner et al. 2007). In a previous paper (Nomura \& Millar 2005, hereafter Paper I), we constructed a model for molecular hydrogen emission from a protoplanetary disk that is irradiated by strong ultraviolet (UV) radiation from a central star, and whose dust component has the same properties as dense molecular cloud dust.

Now, it is known observationally that many young stellar objects emit strong X-ray radiation (Koyama et al. 1994; Feigelson

\footnotetext{
${ }^{1}$ Department of Earth and Planetary Sciences, Kobe University, Nada, Kobe 657-8501, Japan.

2 Astrophysics Research Centre, School of Mathematics and Physics, Queen's University Belfast, Belfast BT7 1NN, Northern Ireland, UK; h.nomura@qub.ac.uk.

${ }^{3}$ Department of Physics, Rikkyo University, Toshima, Tokyo 171-8501, Japan.
}

\& Montmerle 1999; Tsujimoto et al. 2002; Imanishi et al. 2003; Getman et al. 2005; Preibisch et al. 2005) that ionizes hydrogen gas and could be one of the important heating sources of gas in protoplanetary disks, in addition to the grain photoelectric heating induced by far-ultraviolet (FUV) radiation from the central star (Kamp \& Dullemond 2004; Jonkheid et al. 2004; Dullemond et al. 2007; Paper I). Actually, some model calculations show that $\mathrm{X}$-ray irradiation can heat the gas to very high temperatures at the surface layer of the disks (Glassgold \& Najita 2001; Gorti \& Hollenbach 2004; Glassgold et al. 2004; Kamp et al. 2005). Furthermore, it has been suggested that fast secondary electrons produced by X-ray ionization, similar to the electrons induced by cosmic-ray ionization, can pump molecular hydrogen into excited electronic states and may be important, for example, in extragalactic objects, Herbig-Haro objects, and supernova remnants (e.g., Shemansky et al. 1985; Gredel et al. 1989; Gredel \& Dalgarno 1995; Tiné et al. 1997). This X-ray pumping could be observable toward disks that are irradiated by strong X-ray radiation from their central stars (e.g., Bergin et al. 2004).

As disks evolve, it is believed that the dust particles in the disks coagulate and settle toward the disk midplane as the first step of planet formation. The dust dynamics in this stage has been studied theoretically in many works (e.g., Weidenschilling 1980, 1997; Nakagawa et al. 1981, 1986; Mizuno et al. 1988; Mizuno 1989; Cuzzi et al. 1993; Schmitt et al. 1997; Nomura \& Nakagawa 2006). This dust evolution is expected to affect observational properties of the disks, and some model calculations have been done in order to study the effect (e.g., Miyake \& Nakagawa 1993, 1995; D'Alessio et al. 2001, 2006; Dullemond \& Dominik 2004; Jonkheid et al. 2004, 2006, 2007; Rettig et al. 2006; Aikawa $\&$ Nomura 2006). In addition, some numerical calculations of the dust evolution have been done by solving the coagulation equation for settling dust particles, and its effect on the spectral energy 
distribution (SED) of thermal dust emission disks has been investigated (Suttner \& Yorke 2001; Tanaka et al. 2005; Dullemond \& Dominik 2005). In this paper we further examine the effects of the dust evolution on the physical structure of the gas in the disks and on molecular hydrogen emission by using both a simple dust model and a numerical calculation of the coagulation equation.

Historically, line emission from molecular hydrogen has been observed toward various kinds of astronomical objects, such as shock fronts associated with star-forming regions, reflection nebulae, planetary nebulae, supernova remnants, external galaxies, and so on (e.g., Beckwith et al. 1978; Brown et al. 1983; Hasegawa et al. 1987; Burton et al. 1992). The observed line ratios probe the physical properties of these objects as they reflect the excitation mechanisms of molecular hydrogen, e.g., thermal collisions, UV and X-ray pumping, and formation pumping (e.g., Black \& van Dishoeck 1987; Sternberg \& Dalgarno 1989; Tanaka et al. 1989; Tiné et al. 1997; Takahashi \& Uehara 2001). In this work we propose a possible observational diagnostic of the dust evolution in protoplanetary disks using line spectra and the line ratios of molecular hydrogen.

In the following sections we model the density and temperature profiles of the gas and dust in protoplanetary disks, taking into account the X-ray and UV irradiation from a central star, as well as dust growth and settling toward the disk midplane. Then, using the physical structure, we calculate the level populations and line emission of molecular hydrogen. In $\S 2$ we introduce the models we use in this work. For the dust evolution, we use both a simple model and a more realistic model in which we solve the coagulation equation for settling dust particles $(\S 2.1)$. The X-ray and UV radiation fields are computed $1+1$ dimensionally $(\S 2.2)$, and the density and temperature profiles of the gas and dust in the disks are obtained by assuming vertical hydrostatic equilibrium and local thermal and radiative equilibrium ( $§ 2.3)$. The level populations of molecular hydrogen are calculated under an assumption of statistical equilibrium from which we get the molecular hydrogen emission by solving the radiative transfer equation $(\S 2.4)$. In $\S 3$ we present the resulting dust size and spatial distributions ( $(3.1)$, the physical structure of the disks $(\S 3.2)$, the level populations of molecular hydrogen $(\S 3.3)$, and the line spectra and line ratios of molecular hydrogen $(\S 3.4)$, in which the effects of the X-ray irradiation and the dust evolution are discussed. Finally, the results are summarized in $\S 4$.

\section{MODELS}

\subsection{Spatial and Size Distributions of Dust Particles}

The physical structure and the molecular hydrogen emission of the disks are affected by the dust model in various ways: for example, the UV radiation field through dust extinction ( $§ 2.2)$, the dust temperature through optical properties of dust grains, the gas temperature through grain photoelectric heating and dustgas collisions ( $(2.3)$, and the molecular hydrogen formation rate on dust grains $(\S 2.4)$. In this paper we use the following two types of model for the dust size and spatial distributions. In model A we adopt a very simple assumption in order to understand the basic properties of the effects of dust evolution. In model B we consider a more realistic case by numerically solving the coagulation equation for settling dust particles.

In both models the shape of the dust particles is simply assumed to be a compact sphere (for fractal dust aggregate models see, e.g., Kozasa et al. 1992; Ossenkopf 1993; Ormel et al. 2007). Making use of the resulting spatial and size distributions of dust grains, the dust absorption $\left(\kappa_{\nu}\right)$ and scattering $\left(\sigma_{\nu}\right)$ coefficients at each position of the disk are computed by means of the Mie theory (Bohren
\& Huffman 1983), with the dust particles assumed to consist of silicate, carboneous grains, and water ice (for details see Paper I).

\subsubsection{Model $A$}

In model A we assume that the dust grains have a spatially uniform distribution and the mass fractional abundance of the dust with respect to the gas is fixed at each position in the disk (i.e., the dust grains are well mixed with the gas). The size distribution is set to be $d n / d a \propto a^{-3.5}$ ( $a$ is the radius of the dust grain) with the maximum grain radii of $a_{\max }=10 \mu \mathrm{m}, 1 \mathrm{~mm}$, and $10 \mathrm{~cm}$. The minimum radius is set to be $0.01 \mu \mathrm{m}$ for all models. The amount of small dust grains decreases with increasing maximum grain radii as we keep the mass fractional abundance of the dust grains to the gas fixed (e.g., Miyake \& Nakagawa 1993; D’Alessio et al. 2001; Aikawa \& Nomura 2006; see also $\S 2.1 .3$ ).

\subsubsection{Model B}

In this model the spatial and size distributions of dust grains are obtained by solving coagulation equations for various sizes of settling dust particles,

$$
\begin{aligned}
\frac{\partial \varphi(i)}{\partial t}+\frac{\partial}{\partial z}\left[V_{z}(i) \varphi(i)\right]= & -m_{i} \varphi(i) \sum_{j=1}^{n} \beta(i, j) \varphi(j) \\
& +\frac{1}{2} m_{i} \sum_{j=1}^{i-1} \beta(i-j, j) \varphi(i-j) \varphi(j)
\end{aligned}
$$

where $\varphi(i)$ is the mass density of dust particles in a mass bin $i$ whose summation is equal to the total mass density of the dust particles at a given position and time as

$$
\rho_{\text {dust }}(x, z, t)=\sum_{i=1}^{n} \varphi(x, z, t, i) .
$$

Here we briefly summarize the dust evolution model; more details can be found in Nomura \& Nakagawa (2006). Now, $V_{z}(i)$ in equation (1) is the vertical velocity and $m_{i}$ is the typical mass of a particle in a mass bin $i$. The symbol $\beta(i, j)$ is related to the sticking rate of two colliding dust particles, given by

$$
\beta(i, j)=\pi\left(a_{i}+a_{j}\right)^{2} \delta V p_{s} / m_{i} m_{j},
$$

where $a_{i}$ is the radius of a dust particle in a mass bin $i$, and we simply assume the sticking probability of $p_{s}=1$ in this paper. The fragmentation of dust particles is simply neglected in this work. For the relative velocity between two colliding particles, $\delta V$, we adopt

$$
\delta V=\left(\delta V_{\mathrm{B}}^{2}+\delta V_{z}^{2}+\delta V_{x}^{2}+\delta V_{T}^{2}\right)^{1 / 2} .
$$

The symbol $\delta V_{\mathrm{B}}=\left(8 k T_{d} / \pi\right)^{1 / 2}\left(1 / m_{i}+1 / m_{j}\right)^{1 / 2}(k$ is Boltzmann's constant and $T_{d}$ is the dust temperature) is the relative velocity caused by the thermal Brownian motion. The symbols $\delta V_{z}=$ $V_{z}(i)-V_{z}(j)$ and $\delta V_{x}=V_{x}(i)-V_{x}(j)\left[V_{x}(i)\right.$ is the local radial velocity component of dust particles with mass $m_{i}$, arising from angular momentum loss via gas-dust friction] are the velocity differences in the vertical and radial directions, respectively. Finally, $\delta V_{T}$ is the turbulence-induced relative velocity (for more details see Nomura \& Nakagawa 2006). The disk is simply assumed to be completely quiescent or turbulent, with $\delta V_{T}=0$ in a quiescent disk model. 
The mass flux in equation (1) is given by

$$
V_{z}(i) \varphi(i)=-\frac{\Omega_{\mathrm{K}}^{2} z}{A \rho} \varphi(i)
$$

in a quiescent disk, where $\Omega_{\mathrm{K}}$ is the Keplerian frequency and $\rho$ is the gas density. For the drag coefficient between the gas and dust particles, $A$, we adopt $A=c_{S} / \rho_{s} a$ for $a \lesssim l_{g}$ and $A=$ $3 c_{s} l_{g} / 2 \rho_{s} a^{2}$ for $a \gtrsim l_{g}$, following Epstein's and Stokes's laws, respectively, where $c_{s}, \rho_{s}$, and $l_{q}$ are the sound speed of the gas, the solid density of a dust particle, and the mean free path of gas particles, respectively. The mean velocity of the dust particles in the vertical direction, $V_{z}(i)=\Omega_{\mathrm{K}}^{2} z / A \rho$, is obtained by balancing the gas-dust friction force, $A \rho V_{z}(i)$, and the gravitational force in the vertical direction, $\Omega_{\mathrm{K}}^{2} z$. In a turbulent disk, the mass flux is written as

$$
V_{z}(i) \varphi(i)=-\frac{\Omega_{\mathrm{K}}^{2} z}{A \rho} \varphi(i)-D_{0} \rho \frac{\partial[\varphi(i) / \rho]}{\partial z},
$$

following the gradient diffusion hypothesis. For the turbulent diffusivity, we adopt $D_{0}=\alpha^{\prime} c_{s} H /\left(1+\Omega_{\mathrm{K}} / A \rho\right)$, where $H=c_{s 0} / \Omega_{\mathrm{K}}$ ( $c_{S 0}$ is the sound speed at the disk midplane) is the scale height of the disk and we set $\alpha^{\prime}=10^{-4}$ in this paper.

The global radial motion of the dust particles toward the central star is not taken into account in this work. At the disk surface this simplified treatment is applicable because large dust particles settle toward the disk midplane more rapidly than they move toward the central star (Figs. 3 and 4 in $\S 3.1$ actually show that large particles cannot stay in the surface layer). We note that the radial motion is negligible for the small dust particles that couple with the gas efficiently via friction force (e.g., Adachi et al. 1976; Weidenschilling 1977; Takeuchi \& Lin 2005). Therefore, neglect of radial global motion will not affect our results of molecular hydrogen lines that are mainly emitted in the disk surface (see Paper I). In a completely quiescent disk the radial motion is negligible all over the disk (e.g., Nakagawa et al. 1986). Now, in order to avoid making very large particles that do not couple with the gas and should fall on toward the central star, we remove particles larger than some critical radius by simply assuming that when the dust particles grow large enough so that they cannot be trapped in a turbulent eddy, they gain very rapid radial motion. The critical radius is estimated as $a_{\text {crit }}=c_{s} \rho_{\text {gas }} / \rho_{s} \Omega_{\mathrm{K}}$ for $a \lesssim l_{g}$ and $a_{\text {crit }}=$ $\left(3 c_{s} \rho_{\text {gas }} l_{g} / 2 \rho_{s} \Omega_{\mathrm{K}}\right)^{1 / 2}$ for $a \gtrsim l_{g}$ by comparing the friction time between the gas and dust particles, $\tau_{f}=1 / A \rho$, and the turnover time of the largest turbulent eddy, $\tau_{\text {eddy }}=1 / \Omega_{\mathrm{K}}$. We note that the dust particles grow to be larger than $a_{\text {crit }}$ only close to the midplane of a turbulent disk (see $\S 3.1$ ).

As the initial condition of the calculations, we set the dust particles to be well mixed with the gas and the dust-to-gas mass ratio to be spatially uniform. For the initial size distribution of the dust grains, we adopt dust model A with $a_{\max }=10 \mu \mathrm{m}$, which is similar to the dust model of dense molecular clouds (e.g., Weingartner $\&$ Draine 2001). We also assume that the disk is surrounded by a dense molecular cloud with gas number density $n_{\text {out }}=10^{4} \mathrm{~cm}^{-3}$ and consider a continuous input of dust particles from the molecular clouds to the disk as a boundary condition at the disk surface ( $z=z_{\text {coag }}$; see below and $\S 3.1$ ) for the calculations of dust evolution (eq. [1]). Dust particles fall on to the disk because the pressure gradient force is negligibly small for them (e.g., Landau et al. 1967) and cannot sustain them against the gravitational force of the central star (we note that the gas is assumed to be stationary due to the pressure gradient force). This input of dust particles has great influence on structure of the disk surface and observational properties of the disk (see $\S 3$ ). The total mass of dust particles infalling from the cloud to the disk in $10^{6} \mathrm{yr}$ (the time for which the calculations are performed) is $\sim 5 \times 10^{-5} M_{\odot}$, which corresponds to a dust mass in a spherical cloud with a radius of $2500 \mathrm{AU}$ and a gas density of $10^{4} \mathrm{~cm}^{-3}$, and $\frac{1}{3}$ of the initial mass of dust grains in the disk. In the calculations for the dust coagulation and settling we set 23 radial grids logarithmically for $x=0.2-100$ AU and 50 vertical grids for the region where the coagulation becomes significant $\left(z<z_{\text {coag }}\right.$; see $\left.\S 3.1\right)$. The dust coagulation equation is solved using fixed density and temperature profiles of dust and gas because self-consistent calculations coupled to the time evolution of the dust and gas profiles are very time consuming. Instead, we get the dust distribution and the temperature and density profiles by iterating the calculations only once under an assumption that the temperature and density profiles turn into an equilibrium state very quickly; that is, we (1) calculate the dust gas profiles using the initial dust distribution, (2) solve the coagulation equation using the temperature and density profile in step 1, (3) compute the dust and gas profiles again using the dust distribution in step 2 , (4) obtain the dust distribution by solving the coagulation equation with the temperature and density profiles in step 3 , and (5) finally get the dust and gas profiles using the dust distribution in step 4. In the calculations in $\S 3$ we compare the gas temperature and density profiles in steps 3 and 5 and checked that the errors are within $30 \%$ for the gas temperatures and $80 \%$ at most for the gas densities in both quiescent and turbulent disks. These errors are relatively large at the upper layer of the disks where the molecular hydrogen is photodissociated via UV radiation from the central star, so the errors in molecular hydrogen emission from the disks (see $\S 3.4$ ) are smaller and within $15 \%$ for all lines.

\subsubsection{Parameter for Total Surface Area of Dust Particles, $f_{\text {dust }}$}

We introduce a parameter, $f_{\text {dust }}$, that represents the total surface area of dust particles per unit volume of the gas at each position in the disk $(x, z)$,

$$
f_{\text {dust }}(x, z)=A_{\text {tot }}(x, z) / A_{\text {tot }, 0},
$$

where

$$
A_{\text {tot }}(x, z)=\int 4 \pi a^{2} \frac{d n(x, z)}{d a} d a .
$$

The size distributions $d n / d a \propto \varphi(i) / a_{i}^{4}$ in dust model B, and $A_{\text {tot. } 0}$ is calculated using dust model A with $a_{\max }=10 \mu \mathrm{m}$, which is similar to the dust model of dense molecular clouds and is used as the initial condition for dust model B. This parameter controls the physical disk structure and molecular hydrogen emission from the disks because the grain opacity, the grain photoelectric heating rate, and the formation rate of molecular hydrogen on grain surface are roughly proportional to it. Thus, the dust and gas temperatures, the UV radiation field, and the abundance of molecular hydrogen are related to $f_{\text {dust }}$ (see the following subsections). In dust model $\mathrm{A}$, the parameter $f_{\text {dust }}$ has values of $1,0.1$, and 0.01 for the maximum dust size of $a_{\max }=10 \mu \mathrm{m}, 1 \mathrm{~mm}$, and $10 \mathrm{~cm}$, respectively. In model $\mathrm{B}, f_{\text {dust }}$ decreases with dust size growth and settling (except at the disk midplane), which leads to a decrease of the grain photoelectric heating rate, and thus the gas temperature, and so on (see $\S 3.1$ for more details).

\subsection{X-Ray and Ultraviolet Radiation Fields}

Observations have shown that many T Tauri stars emit strong X-ray (e.g., Koyama et al. 1994; Feigelson \& Montmerle 1999; 


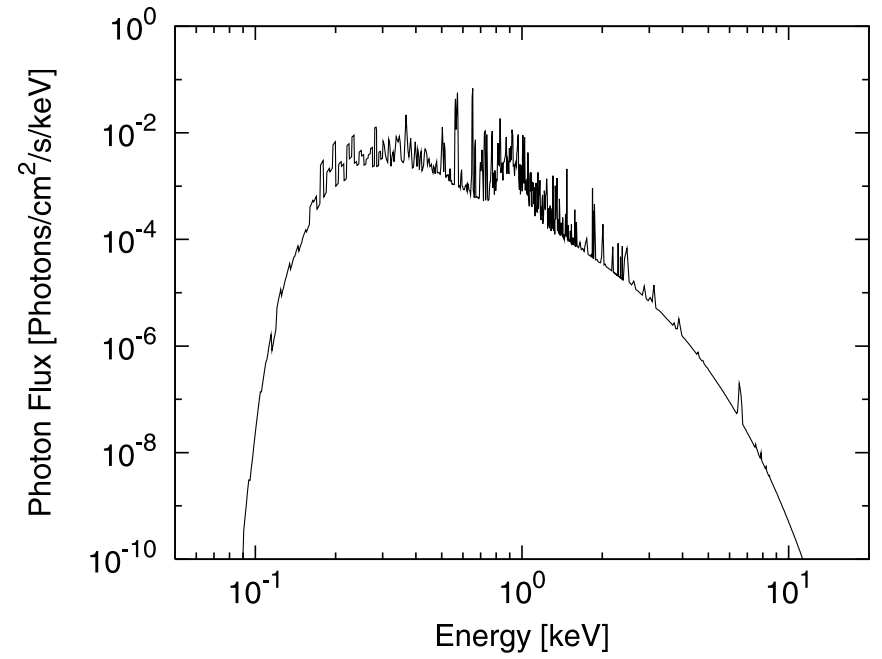

FIG. 1.- Model spectra of the X-ray radiation at the central star, which reproduces observations toward a CTTS, TW Hya $(d=56 \mathrm{pc})$.

Tsujimoto et al. 2002; Imanishi et al. 2003; Getman et al. 2005; Preibisch et al. 2005) as well as strong UV radiation (e.g., Herbig \& Goodrich 1986; Herbst et al. 1994; Valenti et al. 2000). For the $\mathrm{X}$-ray radiation from the central star we use a model that reproduces observational data toward a classical T Tauri star (CTTS), TW Hydrae (e.g., Kastner et al. 2002; Stelzer \& Schmitt 2004). Retrieving the archived XMM-Newton data, we fit the spectrum with a two-temperature thin thermal plasma model (MEKAL model; Mewe et al. 1985; Kaastra 1992; Liedahl et al. 1995), which is often used in order to reproduce the observed X-ray spectrum of $\mathrm{T}$ Tauri stars. The derived best-fit parameters are $k T_{1}=0.8 \mathrm{keV}$ and $k T_{2}=0.2 \mathrm{keV}$ for the plasma temperatures and $N_{\mathrm{H}}=2.7 \times 10^{20} \mathrm{~cm}^{-2}$ for the foreground interstellar hydrogen column density. The total X-ray luminosity of the spectrum corresponds to $L_{\mathrm{X}} \sim 10^{30} \mathrm{ergs} \mathrm{s}^{-1}$. In Figure 1 the resulting model spectra are plotted. The adopted stellar UV radiation field model is also based on observations toward TW Hydrae and analyses by Herbst et al. (1994), Costa et al. (2000), Bergin et al. (2003), Herczeg et al. (2002), and Ardila et al. (2002). The model consists of photospheric blackbody radiation, hydrogenic thermal bremsstrahlung radiation, and strong $\mathrm{Ly} \alpha$ line emission (see Appendix $\mathrm{C}$ of Paper I). The total FUV ( $6 \mathrm{eV}<h \nu<13 \mathrm{eV})$ luminosity corresponds to $L_{\mathrm{FUV}} \sim 10^{31} \mathrm{ergs}^{-1}$. The interstellar $\mathrm{UV}$ radiation field is taken into account, but its contribution is negligible under the strong UV irradiation from the central star (see Paper I for details of the UV radiation field in the disk). We note that although we use the X-ray and UV radiation of TW Hya (as it is one of the most well-observed T Tauri stars), our disk model is to be more widely applicable.

The X-ray and UV fields in the disk are calculated in 1+1 dimensions in the radial and vertical directions (see also Paper I) as

$$
\begin{gathered}
F_{\nu, R}(R, \Theta)=f F_{\nu, \operatorname{star}} \exp \left(-\tau_{\nu, R}\right), \quad \tau_{\nu, R}=\int_{R_{*}}^{R} \chi_{\nu} \rho d R \\
F_{\nu, z}(x, z)=F_{\nu, \text { ISRF }} \exp \left[-\tau_{\nu, z}\left(z_{\infty}\right)\right] \\
+2 \pi \int_{z}^{z_{\infty}} \sigma_{\nu}\left(x, z^{\prime}\right) \rho\left(x, z^{\prime}\right) F_{\nu, R}\left(x, z^{\prime}\right) e^{-\tau_{\nu, z}\left(z^{\prime}\right)} d z^{\prime} \\
\tau_{\nu, z}\left(z^{\prime}\right)=\int_{z}^{z^{\prime}} \chi_{\nu} \rho d z^{\prime \prime}
\end{gathered}
$$

where the direct radiation from the central star is calculated fully, while a plane-parallel approximation in the vertical direction is adopted for the calculation of the scattering process, which could result in overestimating the radiation fields in the disks. In the above equations $F_{\nu \text {, star }}$ is the specific radiation field at the stellar surface and $f=\left(R_{*} / R\right)^{2}$ accounts for the geometrical dilution of the radiation field. $F_{\nu, \mathrm{ISRF}}$ is the FUV interstellar radiation field, and we set $F_{\mathrm{X} \text {-ray, ISRF }}=0$ in the calculation of the X-ray radiation field. The symbols $\tau_{\nu, R}$ and $\tau_{\nu, z}$ are the specific optical depths from the stellar surface $\left(R_{*}, \Theta\right)$ to a point $(R, \Theta)$ and from a point $(x, z)$ to $\left(x, z^{\prime}\right)$, respectively. The symbol $\rho$ is the gas density, and $\chi_{\nu}$ is the monochromatic extinction coefficient defined by the absorption $\left(\kappa_{\nu}\right)$ and scattering $\left(\sigma_{\nu}\right)$ coefficients as $\chi_{\nu} \equiv \kappa_{\nu}+\sigma_{\nu}$. In order to treat X-ray extinction, we adopt the attenuation cross section at an energy $E$ of $\sigma_{\text {att }}(E)=$ $\sigma_{\mathrm{ph}}(E)+\sigma_{\mathrm{Com}}(E)$, where $\sigma_{\mathrm{ph}}$ is the total photoionization cross section due to all elements per hydrogen nucleus and $\sigma_{\mathrm{Com}}$ is the incoherent Compton scattering cross section of hydrogen. For the cross section $\sigma_{\text {ph }}$ we adopt a broken power-law model given by Maloney et al. (1996; see also Wilms et al. 2000), and $\sigma_{\text {Com }}$ is calculated based on McMaster et al. (1969). In calculating equations (9) and (10), we adopt $\chi_{\mathrm{X} \text {-ray }}=\sigma_{\text {att }}(E) / m_{p}$ and $\sigma_{\mathrm{X} \text {-ray }}=$ $\sigma_{\text {Com }}(E) / m_{p}$, where $m_{p}$ is the proton mass.

\subsection{Physical Structure of the Disks}

We model an axisymmetric disk surrounding a central star with the physical parameters of typical T Tauri stars: a mass of $M_{*}=0.5 M_{\odot}$, a radius of $R_{*}=2 R_{\odot}$, and a temperature of $T_{*}=$ 4000 K (e.g., Kenyon \& Hartmann 1995).

The gas temperature and density distributions of the disk are obtained self-consistently by iteratively solving the equations for hydrostatic equilibrium in the vertical direction and local thermal balance between heating and cooling of gas (see Paper I for details). The vertical hydrostatic equilibrium is represented by an equation,

$$
\frac{d P}{d z}=-\rho \frac{G M_{*} z}{\left(x^{2}+z^{2}\right)^{3 / 2}},
$$

where $G$ is the gravitational constant, and $P$ is the gas pressure given by $P=\rho k T / m_{\mu}$, where $\rho, T, k$, and $m_{\mu}$ are the density and temperature of the gas, Boltzmann's constant, and the mean molecular mass, respectively. The condition $\int_{-z_{\infty}}^{z_{\infty}} \rho(x, z) d z=\Sigma(x)$ is imposed, where we set $\rho\left(x, z_{\infty}\right)=5.0 \times 10^{-19} \mathrm{~g} \mathrm{~cm}^{-3}\left(n_{\text {tot }} \approx\right.$ $3 \times 10^{5} \mathrm{~cm}^{-3}$ ) as the boundary condition. The surface density at a disk radius $x, \Sigma(x)$, is defined by assuming a constantly accreting viscous disk model and equating the gravitational energy release of accreting mass to the thermal heating via viscous dissipation at the disk midplane,

$$
\frac{9}{4} \Sigma \alpha c_{s 0}^{2} \Omega_{\mathrm{K}}=\frac{3 G M_{*} \dot{M}}{4 \pi x^{3}}\left[1-\left(\frac{R_{*}}{x}\right)^{1 / 2}\right],
$$

where $c_{s 0}$ and $\Omega_{\mathrm{K}}=\left(G M_{*} / x^{3}\right)^{1 / 2}$ represent the sound speed at the midplane and the Keplerian frequency, respectively. A viscous parameter of $\alpha=0.01$ and a constant mass accretion rate of $\dot{M}=10^{-8} M_{\odot} \mathrm{yr}^{-1}$ are adopted here.

The gas temperature, $T$, is obtained by assuming detailed energy balance at each position in the disk,

$$
\Gamma_{\mathrm{FUV}}+\Gamma_{\mathrm{X} \text {-ray }}+\Lambda_{\mathrm{gr}}+\Lambda_{\text {line }}=0,
$$

\footnotetext{
${ }^{4}$ See http://cars9.uchicago.edu/ newville/mcbook/.
} 
where we include grain photoelectric heating induced by FUV photons, $\Gamma_{\mathrm{FUV}}$, X-ray heating caused by hydrogen ionization, $\Gamma_{\mathrm{X} \text {-ray }}$, gas-grain collisions, $\Lambda_{\mathrm{gr}}$, and radiative cooling by line transitions, $\Lambda_{\text {line }}$, for the gas heating and cooling processes. The $\mathrm{X}$-ray heating rate, $\Gamma_{\mathrm{X} \text {-ray }}$, is calculated as

$$
\Gamma_{\mathrm{X} \text { - ray }}=n_{\text {tot }} f_{h} H_{\mathrm{X}}
$$

where $n_{\text {tot }}$ is the total number density of hydrogen nuclei and $f_{h}$ is the heating efficiency, namely, the fraction of absorbed energy that goes into heating the gas. We adopt $f_{h}=0.1$ for atomic hydrogen and $f_{h}=0.4$ for molecular hydrogen (Maloney et al. 1996; Gorti \& Hollenbach 2004); $H_{\mathrm{X}}$ is the local X-ray energy deposition rate per particle, given by

$$
H_{\mathrm{X}}=\int_{E_{\min }}^{E_{\mathrm{max}}} \sigma_{\mathrm{ph}}(E) F_{\mathrm{X}}(E) d E,
$$

where $\sigma_{\mathrm{ph}}(E)$ is the total photoionization cross section due to all elements per hydrogen nucleus at energy $E$. The symbol $F_{\mathrm{X}}(E)$ is the X-ray energy flux at each position in the disk, and $E_{\min }=$ $0.1 \mathrm{keV}$ and $E_{\max }=10 \mathrm{keV}$ are adopted for the minimum and maximum energy (see Fig. 1 in $\S 2.2$ ). We note that the viscous heating is not taken into account in the energy balance (eq. [13]) because it is not dominant (at the disk surface) if $\alpha=0.01$ (Glassgold et al. 2004).

For radiative cooling by line transitions, we consider the Ly $\alpha$ transition of atomic hydrogen and the metastable transition of $\mathrm{O}_{\mathrm{I}}(26300)$ in addition to the fine-structure transitions of $\mathrm{O}$ I $(63 \mu \mathrm{m})$ and $\mathrm{C}$ II $(158 \mu \mathrm{m})$ and the rotational transitions of CO. In order to calculate the Ly $\alpha$ line cooling, we make use of the table of level populations of atomic hydrogen for various electron densities and temperatures, given by Storey \& Hummer (1995). The collisional de-excitation rate coefficient is taken from Hollenbach \& McKee (1989) for calculation of O I 26300 line cooling. Paper I gives details of calculations of the $\mathrm{O}$ I and $\mathrm{C}$ II fine structure and $\mathrm{CO}$ rotational transition line cooling.

The spatial and size distributions of dust grains affect the gas temperature through the grain photoelectric heating, $\Gamma_{\mathrm{FUV}}$, and the energy exchange between gas and dust particles through collisions, $\Lambda_{\mathrm{gr}}$. Both rates are roughly proportional to the parameter that represents the total surface area of the dust particles, $f_{\text {dust }}$, given in $\S 2.1 .3$. In this paper we simply set $\Gamma_{\mathrm{FUV}}=f_{\text {dust }} \Gamma_{\mathrm{FUV}, 0}$ and $\Lambda_{\mathrm{gr}}=$ $f_{\text {dust }} \Lambda_{\mathrm{gr}, 0}$, where the heating/cooling rates with subscript " 0 " are calculated by using the models given in Paper I, in which we used the dense cloud dust model (see also Aikawa \& Nomura 2006).

The dust temperature profile is important for determining the disk structure because the gas temperature is well coupled to the dust temperature in the dense region near the midplane of the disks. We obtain the dust temperature by assuming local radiative equilibrium between absorption and reemission of radiation by dust grains at each position in the disk. The intensity is calculated by solving the axisymmetric two-dimensional radiative transfer equation by means of the short characteristic method in spherical coordinates (Dullemond \& Turolla 2000; Nomura 2002). As heating sources, we consider the radiative flux produced by the viscous dissipation ( $\alpha$-viscous model) at the disk midplane and the irradiation from the central star (see Paper I for details). The dust evolution in the disks affects the dust temperature through the change in grain opacity $(\S 2.1)$.

\subsection{Level Populations and Line Emission of Molecular Hydrogen}

In order to obtain the molecular hydrogen emission from the disk, we first calculate the abundance and the level populations of the $X^{1} \Sigma_{g}^{-}$electronic state of molecular hydrogen in a statistical equilibrium state, based on Wagenblast \& Hartquist (1988), as

$$
\begin{aligned}
& n_{l}\left(\mathrm{H}_{2}\right)\left[\sum_{m \neq l}\left(A_{l m}+\beta_{l m}+\gamma_{l m}+\sum_{s} n_{s} C_{l m}^{s}\right)+R_{\mathrm{diss}, l}\right] \\
& +k_{\mathrm{O}+\mathrm{H}_{2}} n(\mathrm{O}) n_{l}\left(\mathrm{H}_{2}\right) \\
& =\sum_{m \neq l} n_{m}\left(\mathrm{H}_{2}\right)\left(A_{m l}+\beta_{m l}+\gamma_{m l}+\sum_{s} n_{s} C_{m l}^{s}\right)+n(\mathrm{H}) R_{\text {form }, l},
\end{aligned}
$$

where $A_{l m}$ is the Einstein $A$-coefficient for spontaneous emission from level $l$ to level $m$ and $C_{l m}^{s}$ is the collisional transition rate with collision partner $s$. The symbol $\beta_{l m}$ represents the effective rate for transition $l \rightarrow m$ via UV pumping followed by radiative cascade, and $R_{\text {diss }, l}$ is the photodissociation rate of hydrogen molecules in level $l$; $R_{\text {form, } l}$ is the effective formation rate of $\mathrm{H}_{2}$ in level $l$ on grain surfaces. The endothermic reaction $\mathrm{O}+\mathrm{H}_{2} \rightarrow$ $\mathrm{OH}+\mathrm{H}$, which destroys molecular hydrogen in high-temperature regions, is also taken into account (for details see Paper I). In addition, we consider the effective transition rate, $\gamma_{l m}$, via X-ray pumping of molecular hydrogen followed by radiative cascade. $\mathrm{X}$-ray irradiation from the central star ionizes the gas to produce photoelectrons and subsequently secondary electrons in the disk. Through collisions they excite molecular hydrogen to singlet and triplet electronic states, followed by radiative cascade down into the ground electronic state (e.g., Gredel \& Dalgarno 1995; Tiné et al. 1997; Bergin et al. 2004). In this paper we simply use the entry efficiency $\alpha_{J_{i}}(v, J)$ from the levels $\left(v=0, J_{i}\right)$ to $(v, J)$ for fractional ionization of $10^{-4}$, tabulated in Tiné et al. (1997), in order to estimate the rate $\gamma_{l m}$ as

$$
\gamma_{l m}=\zeta_{\mathrm{X}} \alpha_{J_{l}}\left(v_{m}, J_{m}\right)
$$

This simplified treatment will not cause significant error at the middle layer of the outer disk where molecular hydrogen lines are mainly emitted (the line fluxes are strong where the UV radiation field is not too strong, the gas temperature is moderately high, and the surface area is large; see Paper I), but a full calculation of X-ray pumping and the subsequent radiative cascade should be done in the future. The symbol $\zeta_{X}$ in equation (17) is the total hydrogen ionization rate given by

$$
\zeta_{\mathrm{X}} \simeq N_{\mathrm{sec}} \int_{E_{\min }}^{E_{\max }} \sigma_{\mathrm{ph}}(E) F_{\mathrm{X}}(E) d E,
$$

where $\sigma_{\mathrm{ph}}$ is the total photoionization cross section due to all elements per hydrogen nucleus and $N_{\text {sec }}$ is the number of secondary ionizations of hydrogen per unit energy produced by primary photoelectrons; we put $N_{\mathrm{sec}}=26 \mathrm{keV}^{-1}$ in this paper (e.g., Verner \& Yakovlev 1995; Maloney et al. 1996; Gorti \& Hollenbach 2004). We note that the effect of interstellar cosmicray ionization is not taken into account in the calculation of the pumping process, but it will not affect the results as the X-ray ionization rate is much higher at the disk surface (see $\S 3.2 .1$ ). Possible reactions induced by X-rays are ignored, and a simple chemical network given in Wagenblast \& Hartquist (1988) (plus the reactions $\mathrm{O}+\mathrm{H}_{2} \rightarrow \mathrm{OH}+\mathrm{H}$ and $\left.\mathrm{OH}+h \nu \rightarrow \mathrm{O}+\mathrm{H}\right)$ is adopted as in Paper I. This neglect will not affect the resulting molecular hydrogen abundance since the photodissociation by UV radiation from the central star or the above-mentioned 

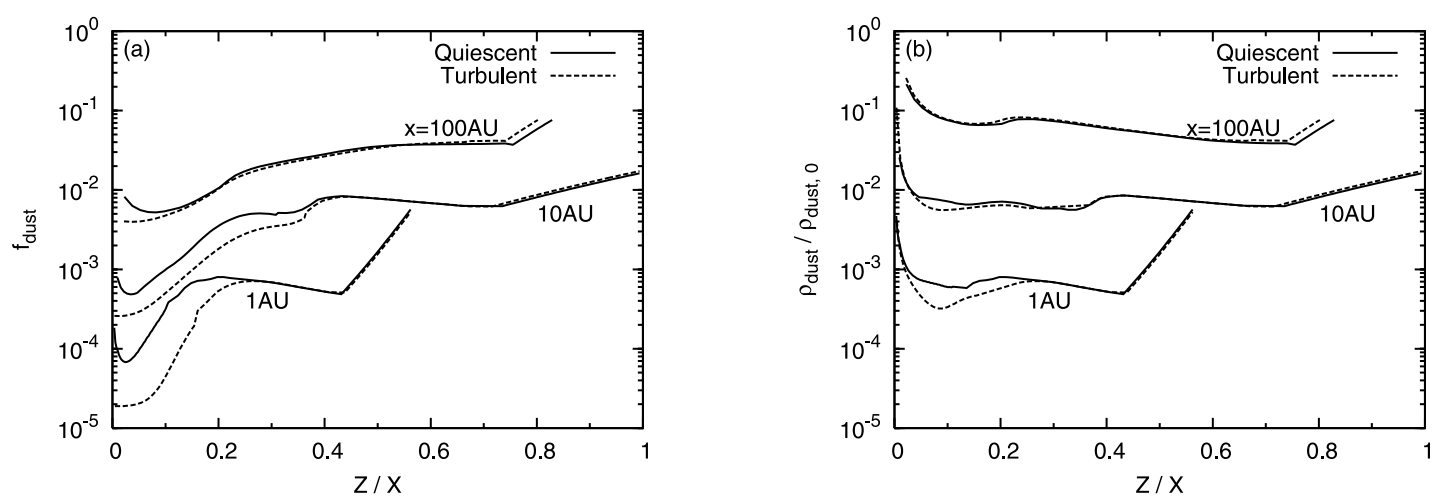

FIG. 2.-Vertical profiles of $(a)$ the parameter for the total surface area of dust grains, $f_{\text {dust }}$, and $(b)$ the total dust density, $\rho_{\text {dust }}$, normalized by the initial value in quiescent (solid lines) and turbulent (dashed lines) disks at the disk radii of $x=1,10$, and $100 \mathrm{AU}$ at $10^{6} \mathrm{yr}$ after the calculations start. At the disk surface $\rho_{\text {dust }} / \rho_{\text {dust }, 0}$ and $f_{\text {dust }}$ are small due to the dust settling toward the disk midplane. Near the disk midplane $f_{\text {dust }}$ is further smaller as a result of the dust coagulation, while $\rho_{\text {dust }} / \rho_{\text {dust }, 0}$ increases due to the dust settling.

reaction with atomic oxygen is more efficient for destroying molecular hydrogen than the X-ray-induced photoionization and other related reactions in our model (for XDR chemistry see, e.g., Maloney et al. 1996; for more detailed disk chemistry, including the X-ray photoprocess, see also, e.g., Aikawa \& Herbst 1999, 2001; Markwick et al. 2002).

The spatial and size distributions of dust grains affect the formation rate of molecular hydrogen. The rate is roughly proportional to the total surface area of the dust particles, $f_{\text {dust }}$, given in $\S 2.1 .3$, so we simply set $R_{\text {form }, l}=f_{\text {dust }} R_{\text {form }, l, 0}$. Here we use the model in Paper I in order to calculate $R_{\text {form } l, 0 .}$. The total formation rate is given by $\sum_{l} R_{\text {form }, l}=7.5 \times 10^{-18} f_{\text {dust }} T^{0.5} \epsilon_{\mathrm{H}_{2}}\left(T_{d}\right) n_{\mathrm{tot}} n(H)$ $\mathrm{cm}^{-3} \mathrm{~s}^{-1}$, where $T$ is the gas temperature and $\epsilon_{\mathrm{H}_{2}}\left(T_{d}\right)$ is the recombination efficiency of atomic hydrogen on dust grains as a function of the dust temperature, $T_{d}$ (Cazaux \& Tielens 2002, 2004; see also Pirronello et al. 1999; Zecho et al. 2002).

Making use of the physical properties obtained in the previous subsections and the level populations, we calculate emission (from levels $u$ to $l$ ) of molecular hydrogen from the disks by integrating the radiative transfer equation (for details see Paper I),

$$
F_{u l}=\frac{1}{4 \pi d^{2}} \int_{x_{\mathrm{in}}}^{x_{\mathrm{out}}} 2 \pi x d x \int_{-z_{\infty}}^{z_{\infty}} \tilde{\eta}_{u l}(x, z) d z
$$

where $\tilde{\eta}_{u l}(x, z)$ is the emissivity of the transition line at $(x, z)$ times the effect of absorption in the upper disk layer, given by

$$
\tilde{\eta}_{u l}(x, z)=n_{u}(x, z) A_{u l} \frac{h \nu_{u l}}{4 \pi} \exp \left[-\tau_{u l}(x, z)\right]
$$

Here $\tau_{u l}(x, z)$ is the optical depth from $z$ to the disk surface $z_{\infty}$ at the frequency $\nu_{u l}$,

$$
\tau_{u l}(x, z)=\int_{z}^{z_{\infty}} \chi_{u l}\left(x, z^{\prime}\right) d z^{\prime}
$$

where $\chi_{u l}$ is the total extinction coefficient,

$$
\chi_{u l}=\rho \chi_{\nu_{u l}}+\left(n_{l} B_{l u}-n_{u} B_{u l}\right) \Phi_{u l} \frac{h \nu_{u l}}{4 \pi} .
$$

In these equations, $A_{u l}$ and $B_{u l}$ are the Einstein coefficients, $n_{u}$ and $n_{l}$ are the number densities of the upper and lower levels, respectively, and $\Phi_{u l}$ is the line profile function. The energy difference between the levels $u$ and $l$ corresponds to $h \nu_{u l}$. The symbol $\chi_{\nu_{u l}}$ is the extinction coefficient of dust grains (see $\S \S 2.1$ and
2.2) at the frequency $\nu_{u l}$, and $\rho$ is the gas density. Here the disk is assumed to be face-on to an observer, and we use the distance to an object of $d=56 \mathrm{pc}$ for calculating the intensity in order to compare it with the observations toward TW Hya. Extinction by foreground interstellar dust grains is not taken into account in the calculations.

\section{RESULTS}

\subsection{Spatial and Size Distributions of Dust Particles}

For dust model B we obtain the spatial and size distributions of dust particles by solving the coagulation equations for various sizes of settling dust particles in a quiescent or turbulent disk $(\S 2.1)$. In Figure 2 we plot the resulting profiles of $(a)$ the parameter representing the total surface area of dust grains, $f_{\text {dust }}$ (defined in eq. [7] in $\S 2.1 .3$ ), and (b) the total dust density $\rho_{\text {dust }}$ normalized by the initial value $\rho_{\text {dust }, 0}$ (defined in eq. [2] in $\S 2.1 .2$ ) in the vertical direction at the disk radii of $x=1,10$, and $100 \mathrm{AU}$. The initial dust density is simply proportional to the gas density (the dust particles are well mixed with the gas) and corresponds to roughly $1 \%$ of the gas mass density, $\rho_{\text {dust }, 0} \approx 0.01 \rho$, in this model. The solid and dashed lines are the profiles in quiescent and turbulent disks, respectively. The calculations are performed for $10^{6} \mathrm{yr}$, comparable to the typical age of CTTSs. We note that at $t \sim 10^{6} \mathrm{yr}$ the dust coagulation process and settling motion (input from the upstream and output to the downstream) are almost in equilibrium state at each position in the disk, and the spatial and size distributions of dust particles do not change with time except in the region very close to the disk midplane. Therefore, the spatial and size distributions of dust particles in the surface layer presented in this subsection are applicable to older star-disk systems as well. Figure 2 shows that the mass and total surface area of dust grains per unit volume of the gas are much smaller than the initial values. In the disk surface $\left(z>z_{\text {coag }}\right.$; see below) where the density of particles is low enough so that the dust particles settle before they grow, $\rho_{\text {dust }} / \rho_{\text {dust }, 0}$ and $f_{\text {dust }}$ are small due to the settling of dust particles toward the disk midplane. In the upper surface of the disk $\left(z>z_{\text {fric }}\right.$; see below), where the density is low enough that the gas friction force does not affect the motion of dust particles, the particles settle in the vertical direction with the free-fall velocity, $V_{z}=V_{\mathrm{ff}}=\left[2 G M_{*} /\left(x^{2}+z^{2}\right)^{1 / 2}\right]^{1 / 2}$. In this region the normalized dust density $\rho_{\text {dust }} / \rho_{\text {dust }, 0}$ (and the parameter $\left.f_{\text {dust }}\right)$ drops with decreasing $z$, inversely proportional to the gas (or the initial dust) density. Here the dust particles are assumed to be continuously falling from the surrounding molecular cloud to the disk due to the gravitational force of the central star with a constant 

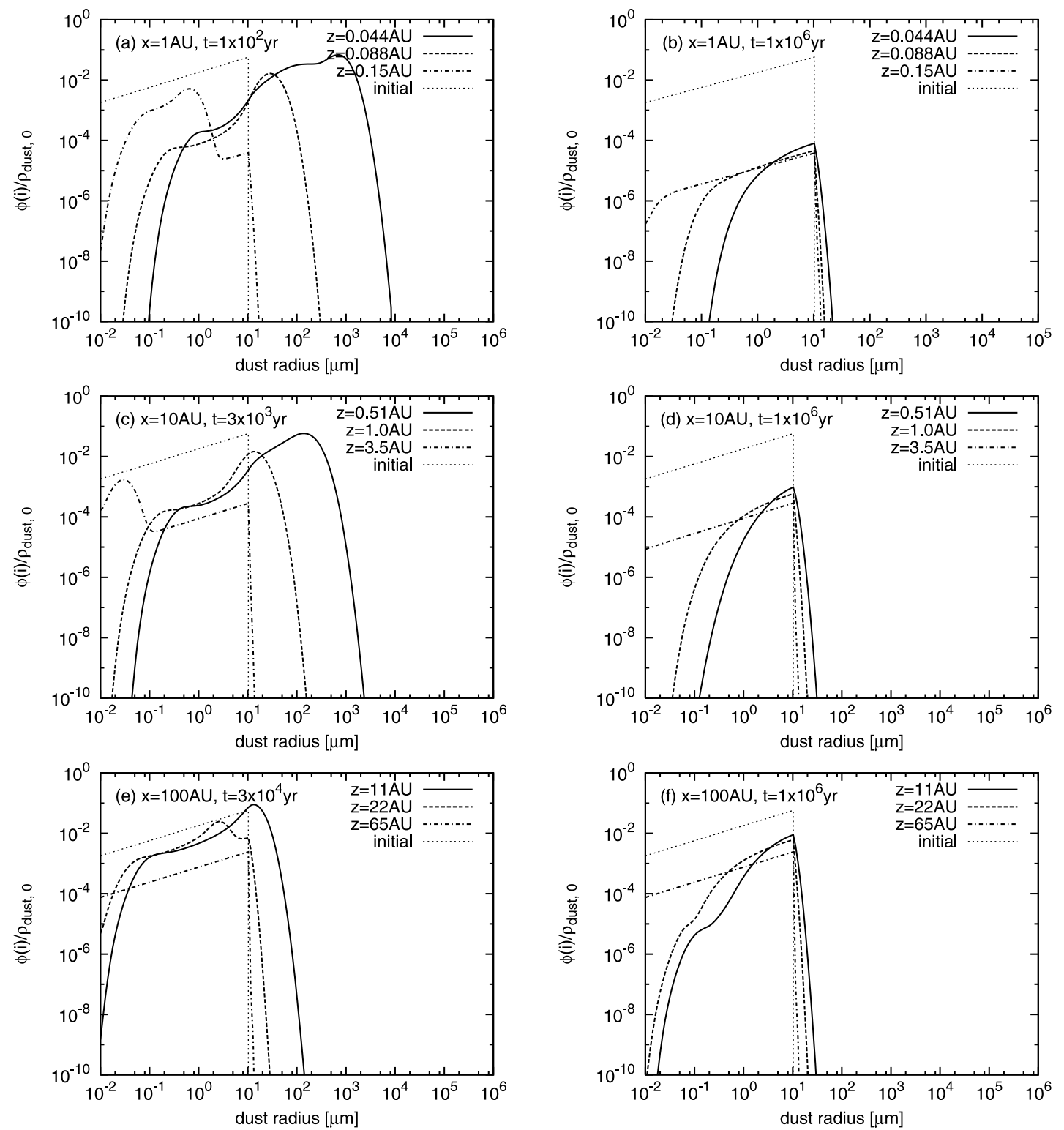

Fig. 3.-Size distributions of mass density of dust particles, $\varphi(i)$, normalized by the initial dust density $\rho_{\text {dust } 0} 0$ at each disk radius, $x$, and time, $t$, in a quiescent disk. The dot-dashed, dashed, and solid lines represent the distributions at $z \sim z_{\text {coag }}, z \sim 2 H$, and $z \sim H$, respectively. The thin dotted lines show the initial distribution. At the disk surface the size distributions are similar to those in molecular clouds, but the number density of the dust particles is much smaller than the initial value due to the dust settling. Near the disk midplane small particles disappear due to the dust coagulation and larger particles settle toward the midplane as time increases.

(time independent) mass flux ( $=n_{\text {out }} V_{\mathrm{ff}} ;$ see $\left.\S 2.1 .2\right)$. At smaller $z\left(z<z_{\text {fric }}\right)$ where the gas density becomes higher and the gas friction force controls the dust motion, the vertical velocity of dust particles becomes $V_{z}=\Omega_{\mathrm{K}}^{2} z / A \rho(\S 2.1 .2)$, and the normalized dust density $\rho_{\text {dust }} / \rho_{\text {dust }, 0}$ (and the parameter $f_{\text {dust }}$ ) does not change very much in this region. The velocity changes from the free-fall velocity $\left(V_{\mathrm{ff}}\right)$ to the terminal velocity $\left(V_{z}=\Omega_{\mathrm{K}}^{2} z / A \rho\right)$ around $z=z_{\text {fric }}=0.5,7.5$, and $75 \mathrm{AU}$ at the disk radii of $x=1$, 10 , and $100 \mathrm{AU}$, respectively, in this model. At even smaller $z\left(z<z_{\text {coag }}\right)$ where the density is much higher and the collisional cross section becomes high enough for the dust particles to grow, the parameter $f_{\text {dust }}$ drops with decreasing $z$ (and increasing density) because small particles disappear as a result of coagulation, while the normalized dust density $\rho_{\text {dust }} / \rho_{\text {dust }, 0}$ does not change very much and increases close to the disk midplane due to the settling of the particles. Most of the dust mass settles at the disk midplane and $\rho_{\text {dust }} / \rho_{\text {dust }, 0} \gg 1$ at $z \approx 0$ (not shown in this figure). Throughout the calculations the total dust mass in the disk is equal to the initial dust mass plus the mass infalled from the cloud (minus the mass of particles with $a>a_{\text {crit }}$ removed near the midplane of the turbulent disk; see below). The difference between the quiescent and turbulent disks shows up most clearly in the parameter $f_{\text {dust }}$ at small $z\left(z<z_{\text {coag }}\right.$; where the dust coagulation is efficient) because the collision rate is higher in the turbulent disk owing to the turbulence-induced relative velocity between the particles, $\delta V_{T}$ (see $\S 2.1 .2$ ). The coagulation becomes efficient around $z=z_{\text {coag }} \sim 0.15(0.2), 3.5(4.0)$, and 65 (65) AU for the quiescent (turbulent) disk at $x=1,10$, and $100 \mathrm{AU}$, respectively, in this model.

In Figures 3 and 4 we plot the resulting size distributions of mass density of dust particles, $\varphi(i)$, normalized by the initial dust density $\rho_{\text {dust, } 0}$, in quiescent and turbulent disks, respectively. Each figure shows the size distributions at $(a) x=1 \mathrm{AU}, t=1 \times 10^{2} \mathrm{yr}$; (b) $x=1 \mathrm{AU}, t=1 \times 10^{6} \mathrm{yr}$; (c) $x=10 \mathrm{AU}, t=3 \times 10^{3} \mathrm{yr}$; (d) $x=10 \mathrm{AU}, t=1 \times 10^{6} \mathrm{yr}$; $(e) x=100 \mathrm{AU}, t=3 \times 10^{4} \mathrm{yr}$; and $(f) x=100 \mathrm{AU}, t=1 \times 10^{6} \mathrm{yr}$. The time used in $(a)$, 

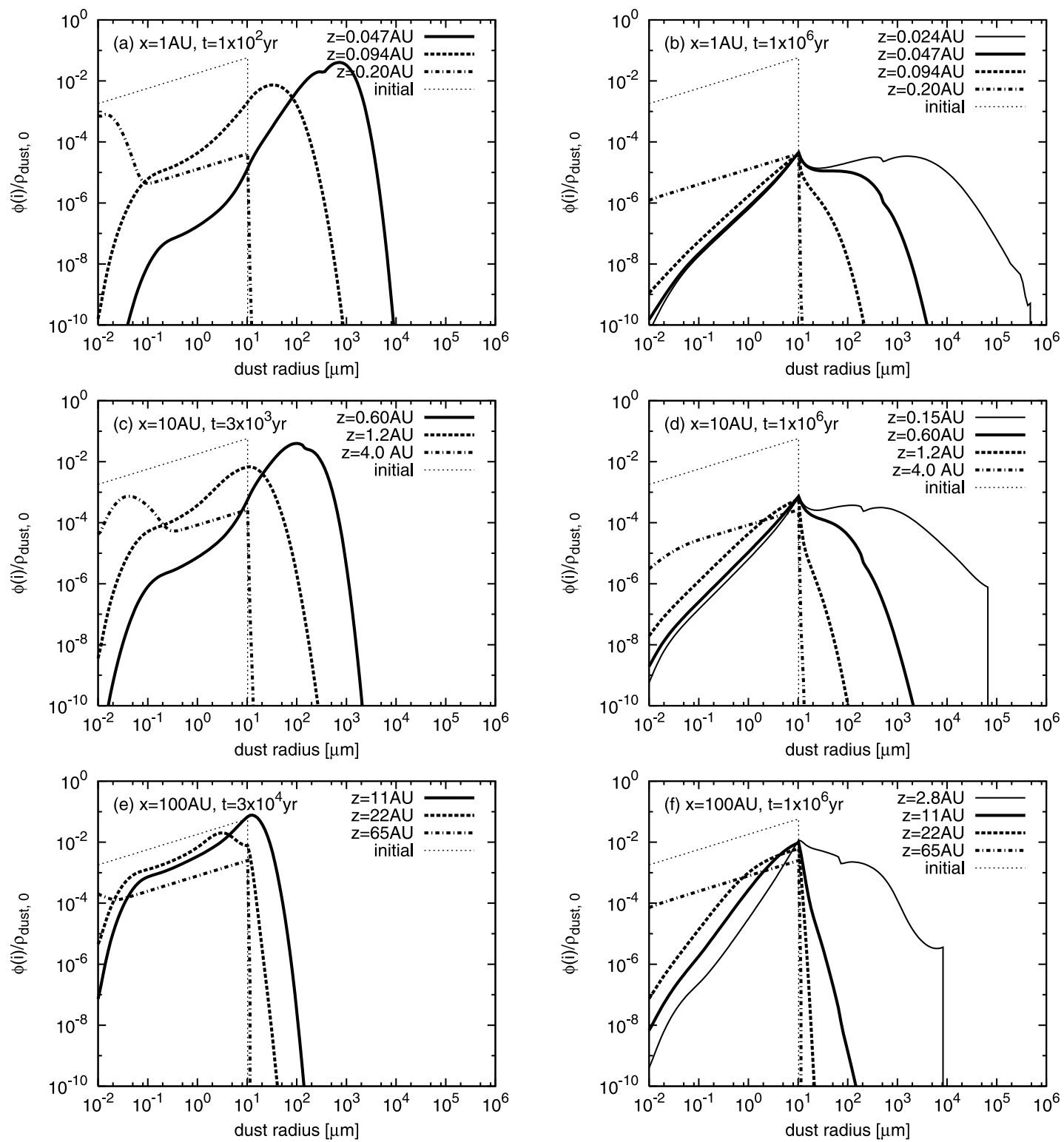

FIg. 4.- Same as Fig. 3, but in a turbulent disk. The size distributions of dust particles at $z \sim 0.25 H$ are also plotted as thin solid lines in $(b),(d)$, and $(f)$. Near the disk midplane a certain number of large dust particles remain due to turbulent mixing.

$(c)$, and $(e)$ is around the time when the size of the largest dust particles at the disk height of $z \sim H$ becomes maximum in the quiescent disk model. The dot-dashed, dashed, and solid lines in each figure represent the size distributions at $z \sim z_{\text {coag }}, z \sim 2 H$, and $z \sim H$, respectively. In Figures $4 b, 4 d$, and $4 f$ we also plot the size distributions at $z \sim 0.25 \mathrm{H}$ as thin solid lines. The disk scale heights are $H=0.044$ (0.047), $0.51(0.60)$, and 11 (11) AU for the quiescent (turbulent) disk at $x=1,10$, and $100 \mathrm{AU}$, respectively. The thin dotted lines show the distribution of the initial condition (dust model A with $a_{\max }=10 \mu \mathrm{m}$ ). The figures show that at the surface layer above $z \sim z_{\text {coag }}$ the size distributions at $t=10^{6} \mathrm{yr}$ are similar to those in dense molecular clouds (dust model A with $a_{\max }=10 \mu \mathrm{m}$ in this work) as the dust particles cannot grow due to the small collisional rate, but the number density of the particles is much smaller than the initial value due to the dust settling as mentioned above. We note that bumps of mass density of small dust particles $(a \lesssim 1 \mu \mathrm{m})$ at $z \sim z_{\text {coag }}$ in early phases are remnants of the initial distribution. At smaller $z(z<$ $z_{\text {coag }}$ ), small dust particles disappear as they stick together to make larger particles. In the quiescent disk the larger particles settle toward the disk midplane and disappear from the disk surface, $z \geq H$, as time goes on (Fig. 3). Meanwhile, in the turbulent disk a certain amount of large particles remain even at $z \geq H$ at $t=10^{6} \mathrm{yr}$ (although most of them settle toward the midplane) because of the turbulent mixing that works so as to unify the size distributions in the vertical direction (§ 2.1.2; Fig. 4). The cutoffs around the dust radii of $a \sim 7$ and $0.8 \mathrm{~cm}$ at the disk height of $z \sim 0.25 H$ in Figures $4 d$ and $4 e$ correspond to the critical radii, $a_{\text {crit }}$, beyond which the particles cannot be trapped in a turbulent eddy and move toward the central star rapidly. The particles with $a>a_{\text {crit }}$ are simply removed from the calculations (see $\S 2.1 .2)$.

\subsection{Physical Properties of the Disks}

In this subsection we obtain the gas density and temperature distributions of the disk self-consistently by iteratively solving the equations for vertical hydrostatic equilibrium and local thermal balance between heating and cooling of gas ( $(2.3)$. The effects of 


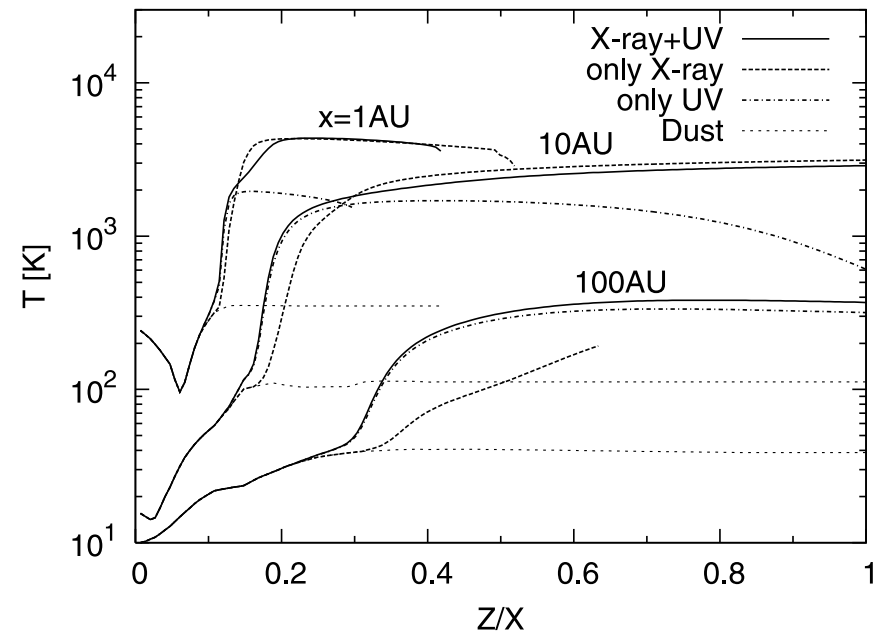

FIG. 5.-Vertical temperature profiles of dust (thin dotted lines) and gas at the disk radii of $x=1,10$, and $100 \mathrm{AU}$ for the irradiation models of X-rays+UV (solid lines), X-rays only (dashed lines), and UV only (dot-dashed lines). Dust model A with $a_{\max }=10 \mu \mathrm{m}$ is used. The X-ray heating is dominant at the inner region and the very surface layer of the disk, while the FUV heating dominates in the middle layer and the outer region of the disk.

the X-ray irradiation from the central star and the dust evolution on the physical properties of the disks are discussed in the following.

\subsubsection{Effect of X-Rays}

First, in Figure 5 we plot the gas temperature profiles in the vertical direction at the disk radii of $x=1,10$, and $100 \mathrm{AU}$, where the disk is irradiated by both X-ray and UV radiation from the central star (solid lines). We also plot the profiles for a disk that is irradiated by X-ray radiation only (dashed lines) or UV radiation only (dot-dashed lines) for comparison. The thin dotted lines are the dust temperature profiles that are not affected by the UV or X-ray irradiation model. Dust model A with the maximum dust radius of $a_{\max }=10 \mu \mathrm{m}$ (see $\left.\S 2.1\right)$ is used throughout this subsection. We note that the calculations are performed in the region where $\rho \geq \rho\left(x, z_{\infty}\right)=5.0 \times 10^{-19} \mathrm{~g} \mathrm{~cm}^{-3}$, and the position of $z_{\infty}$ depends on the models (see $\S 2.3$ ). The figure shows that the gas temperature is much higher than the dust temperature in the surface layer of the disk due to the X-ray and FUV heating. The X-ray heating dominates the FUV heating in the inner region and in the surface layer of the disk where direct irradiation from the central star is strong. Meanwhile, the FUV heating dominates the X-ray heating in the middle layer and in the outer disk. This is because the FUV radiation is scattered efficiently by dust grains, while the Compton scattering of X-ray radiation is inefficient in the energy range of $E \lesssim 1 \mathrm{keV}$ (e.g., Igea \& Glassgold 1999), in which T Tauri stars mainly emit X-rays (see Fig. 1). The gas temperature is almost the same as the dust temperature near the disk midplane, where the density is high enough that the gas and dust particles are well coupled through collisions.

In Figure 6 we plot the vertical profiles of the heating and cooling rates at disk radii of $(a) 1 \mathrm{AU},(b) 10 \mathrm{AU}$, and (c) $100 \mathrm{AU}$, for a disk irradiated by both X-ray and UV radiation from the central star. The figures clearly show that the X-ray heating dominates in the inner region and in the surface layer of the disk, while the FUV heating dominates in the middle layer and the outer region of the disk. With regard to the cooling processes, radiative cooling dominates in the surface layer, while dust-gas collisions dominate near the midplane where the density is high. The main coolant at the surface layer changes as $\mathrm{Ly} \alpha, \mathrm{O}_{\mathrm{I}} \lambda 6300$, and $\mathrm{O}_{\mathrm{I}} 63 \mu \mathrm{m}$ at the
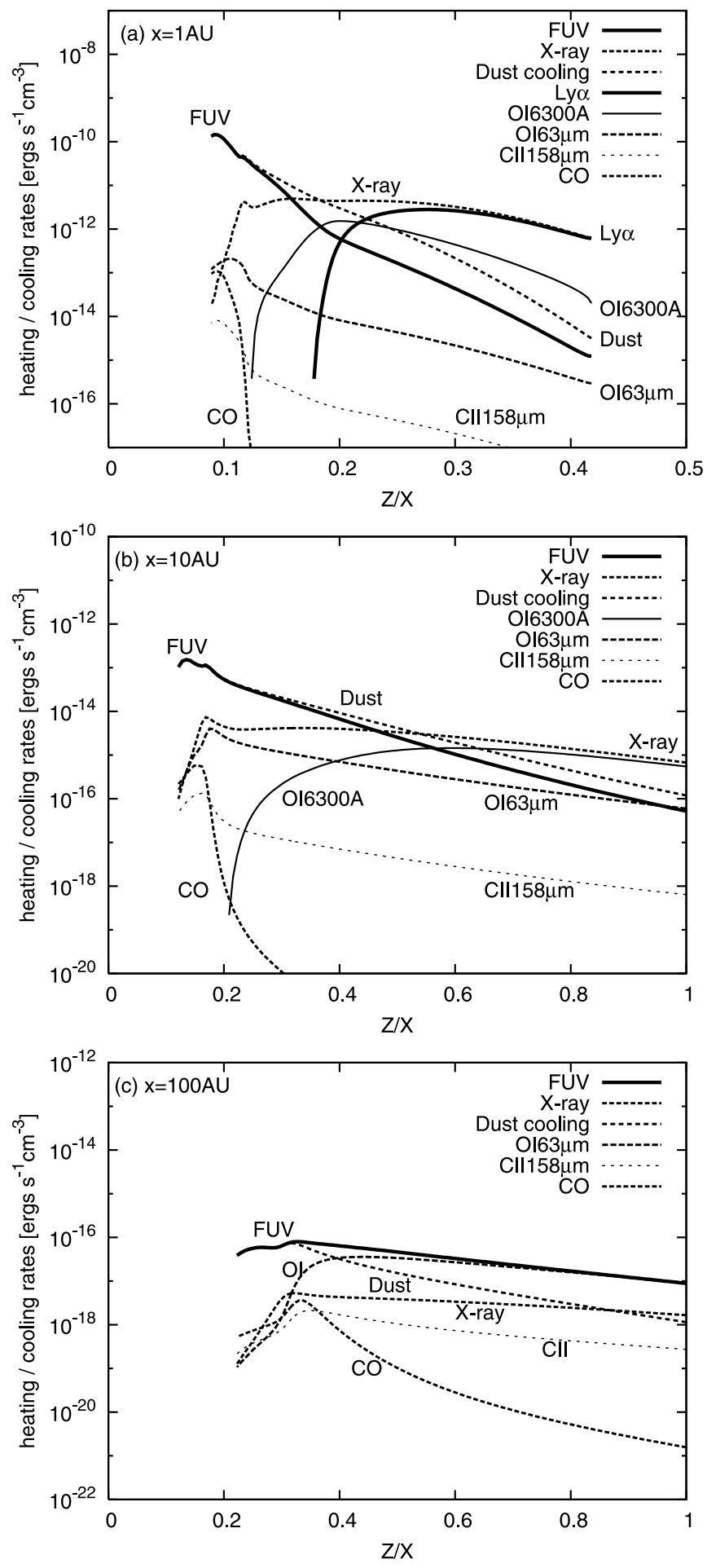

FIG. 6.-Vertical profiles of the cooling and heating rates at the disk radii of (a) $x=1 \mathrm{AU},(b) 10 \mathrm{AU}$, and (c) $100 \mathrm{AU}$ for the irradiation model of X-rays+UV and dust model A with $a_{\max }=10 \mu \mathrm{m}$. The X-ray or FUV heating dominates the heating process, while the radiative cooling (Ly $\alpha$, O I 26300 , and $\mathrm{O}$ I $63 \mu \mathrm{m}$ for $x=1,10$, and $100 \mathrm{AU}$ ) and the dust-gas collision dominate the cooling process at the surface layer and near the midplane, respectively.

disk radii of 1,10 , and $100 \mathrm{AU}$, respectively, with decreasing gas temperature. These properties are qualitatively the same even if we use the different dust models in $\S 2.1$.

Furthermore, we plot in Figure 7 the vertical profiles of the $\mathrm{X}$-ray ionization rates, $\zeta_{\mathrm{x}}$, defined in equation (18), at disk radii of 1,10 , and $100 \mathrm{AU}$, where the disk is irradiated by both X-ray 


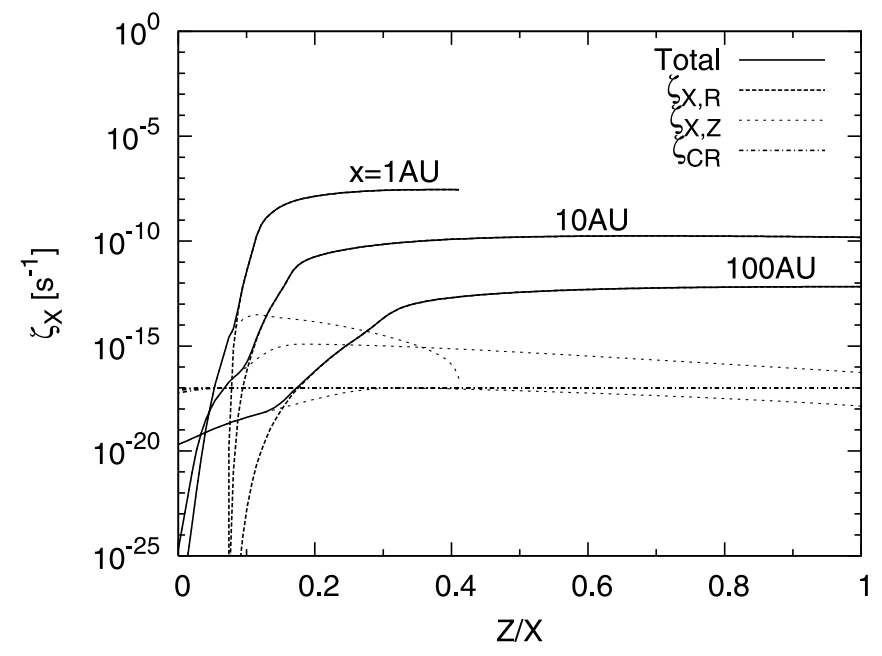

FIG. 7.- Vertical profiles of the X-ray ionization rate at the disk radii of $x=1$, 10 , and $100 \mathrm{AU}$ for the irradiation model of X-rays+UV and dust model A with $a_{\max }=10 \mu \mathrm{m}$. The solid, dashed, and dotted lines show the total rate, radial, and vertical components, respectively. The ionization rate due to interstellar cosmic rays is plotted as a dot-dashed line for comparison. The ionization rates by the $\mathrm{X}$-rays from the central star are much lower than those by the interstellar cosmic rays near the disk midplane due to the inefficient Compton scattering of the X-ray radiation.

and $\mathrm{UV}$ radiation from the central star. The radial $\left(\zeta_{\mathrm{x}, R} ;\right.$ dashed lines $)$ and vertical $\left(\zeta_{\mathrm{X}, z} ;\right.$ dotted lines $)$ components, which are calculated by substituting $F_{\mathrm{X}, R}$ and $F_{\mathrm{X}, z}$ of equations (9) and (10) into equation (18) and satisfy $\zeta_{\mathrm{X}}=\zeta_{\mathrm{X}, R}+\zeta_{\mathrm{X}, z}$, are also plotted for comparison. In addition, the ionization rates caused by interstellar cosmic rays, $\zeta_{\mathrm{CR}}$, are plotted as dot-dashed lines and estimated as

$$
\zeta_{\mathrm{CR}}=\zeta_{\mathrm{CR}, 0} \exp \left[-\Sigma(z) / \chi_{\mathrm{CR}}\right]
$$

where we adopt $\zeta_{\mathrm{CR}, 0}=1 \times 10^{-17} \mathrm{~s}^{-1}$ and the attenuation coefficient of $\chi_{\mathrm{CR}}=96 \mathrm{~g} \mathrm{~cm}^{-2}$ (Umebayashi \& Nakano 1981). The surface density is calculated as $\Sigma(z)=\int_{z}^{z_{\infty}} \rho\left(z^{\prime}\right) d z^{\prime}$. The figure shows that at the disk surface the ionization rates due to $\mathrm{X}$-rays from the central star are much higher than those due to interstellar cosmic rays, while near the disk midplane the former is much lower than the latter. This is because X-ray attenuation is larger than that of cosmic rays and because the Compton scattering of X-ray radiation is inefficient (see Fig. 1 and Igea \& Glassgold 1999).

\subsubsection{Effect of Dust Evolution}

In Figure 8 we plot the vertical gas temperature profiles for various dust models at disk radii of $x=1,10$, and $100 \mathrm{AU}$ for the case of a disk heated by both X-rays and UV radiation. The profiles for dust model A with different maximum dust radii of $a_{\max }=$ $10 \mu \mathrm{m}$ (solid lines), $1 \mathrm{~mm}$ (dashed lines), and $10 \mathrm{~cm}$ (dot-dashed lines) are plotted together in Figure $8 a$. The profiles for dust model B at $10^{6} \mathrm{yr}$ after the calculation starts are plotted in Figure $8 b$ for the quiescent (solid lines) and turbulent (dot-dashed lines) disks. The profiles calculated by using the dense cloud dust model (which is the initial condition of the calculation for the dust evolution and dust model A with $a_{\max }=10 \mu \mathrm{m}$ ) are also plotted together as thin solid lines for comparison. The thin dotted lines in the figures are the dust temperature profiles. Figure $8 a$ shows that as the dust particles grow and the total surface area of dust grains $\left(f_{\text {dust }}\right)$ decreases (see $\S 2.1 .3$ ), the gas temperature at the disk surface drops because the grain photoelectric heating rate decreases (e.g., Aikawa \& Nomura 2006). The gas temperatures in the inner disk $(x \sim 1 \mathrm{AU})$ and in the surface layer at $x \sim 10$ AU do not change because the X-ray heating dominates in these regions. The dust temperature at the disk surface decreases slightly with dust growth. Figure $8 b$ shows that clear differences appear in the gas and dust temperatures between the dust model of dense clouds (dust model A with $a_{\max }=10 \mu \mathrm{m}$ ) and the models with the dust evolution in both quiescent and turbulent disks. For the models with dust evolution the gas temperature in the middle layer and the outer region of the disks drops owing to the decrease of $f_{\text {dust }}$ (see $\S \S 2.1 .3$ and 3.1), while the dust and gas temperatures near the midplane increase because the grain opacity decreases and the irradiation from the central star can penetrate deeper into the disks. At $x \sim 1$ AU heating via irradiation dominates even near the midplane for the models with the dust evolution, whereas the viscous heating is dominant for the dense cloud dust model. The differences between the quiescent and turbulent disks are small because the profiles of $f_{\text {dust }}$ are similar, especially in the surface layer. The dust and gas temperatures very close to the midplane are slightly higher in the turbulent disk due to the higher collision rate between the dust particles, which results in lower $f_{\text {dust }}$ and grain opacity (see $\S 3.1$ ).

Dust growth and settling are also expected to impact on the $\mathrm{X}$-ray heating rates and the gas temperature profile through the change in the photoionization cross section, $\sigma_{\mathrm{ph}}$, part of which is contributed by heavy elements in dust grains (e.g., Glassgold et al. 1997; Wilms et al. 2000). Here we check the effects by simply

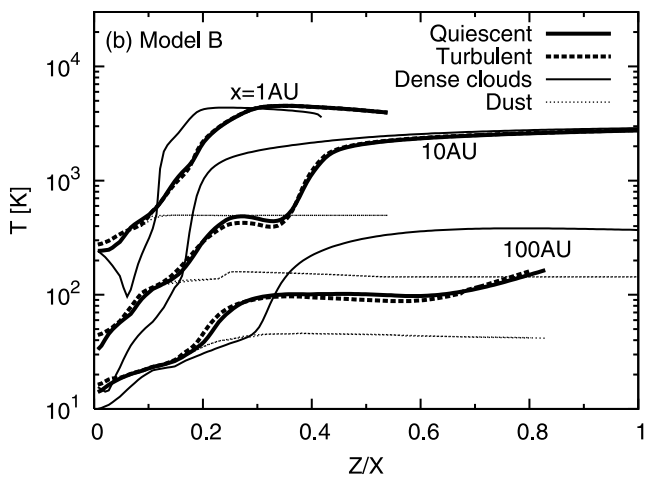

FIG. 8.-Vertical temperature profiles of dust (thin dotted lines) and gas at the disk radii of $x=1,10$, and 100 AU for (a) dust model A with $a_{\text {max }}=10 \mu \mathrm{m}$ (solid lines), $1 \mathrm{~mm}$ (dashed lines), and $10 \mathrm{~cm}$ (dot-dashed lines) and (b) model B in quiescent (solid lines) and turbulent (dashed lines) disks. The profiles for the dense cloud dust model (dust model A with $a_{\max }=10 \mu \mathrm{m}$ ) are plotted here as thin solid lines. The irradiation model of X-rays $+\mathrm{UV}$ is used here. As the dust particles grow or settle toward the disk midplane, the gas temperature in the middle layer and in the outer disk decreases due to the decrease of the grain photoelectric heating rate. Meanwhile, the dust and gas temperatures near the midplane increase due to smaller grain opacity and greater penetration of the irradiation from the central star. 


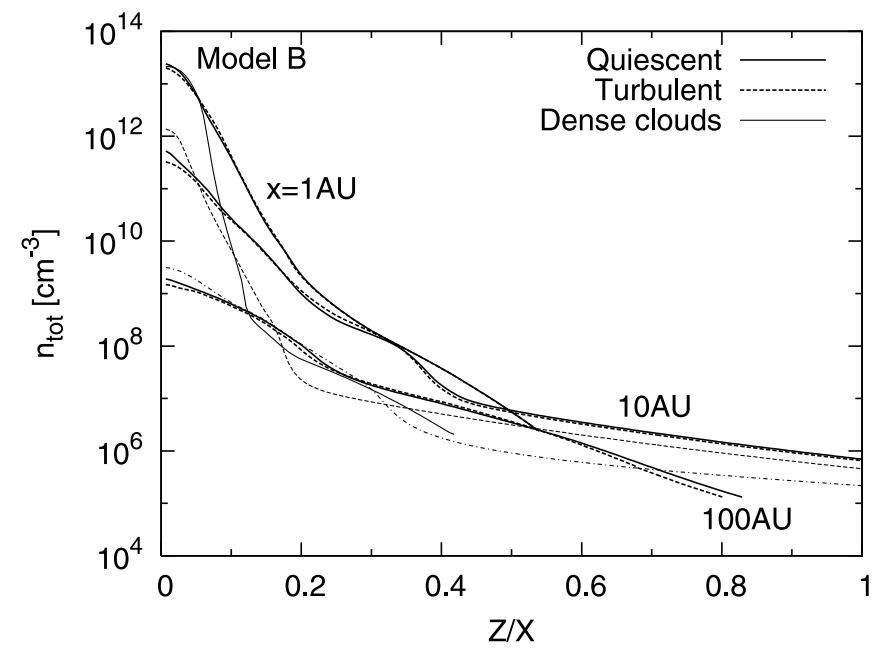

FIG. 9.-Vertical gas density profiles at the disk radii of $x=1,10$, and $100 \mathrm{AU}$ for dust model B in quiescent (solid lines) and turbulent (dashed lines) disks. The profiles for the dense cloud dust model are also plotted together as thin solid $(x=1 \mathrm{AU})$, dotted $(x=10 \mathrm{AU})$, and dot-dashed $(x=100 \mathrm{AU})$ lines. The disks are puffed out more for the models with the dust evolution due to higher gas temperatures at the disk midplane and higher disk scale height.

adopting an extreme case, specifically that the contribution of dust grains to the cross section is negligible for dust model A with $a_{\max }=10 \mathrm{~cm}$ and for dust model B. We modify the cross section in Maloney et al. (1996) by simply assuming that the contribution by heavy elements in gas phase is about $60 \%$, on average, of the total cross section (Wilms et al. 2000). This modification of the cross section makes the gas temperature higher or lower by a factor of 2 at most at the disk surface, where the X-ray heating is dominant. At large $z$ the gas temperature becomes slightly lower due to the decrease of the photoionization rate, while at smaller $z$, where the influence of attenuation is more important, the temperature becomes a bit higher owing to the decrease of the attenuation coefficient, which results in the relatively stronger X-ray radiation field (e.g., Glassgold et al. 1997). The gas density at the disk surface also becomes higher or lower by a factor of 3 at most, according to the change in the gas temperature. The variation of the photoionization cross section due to the dust evolution will also slightly affect the level populations and the line emission of molecular hydrogen through the changes in the thermal collision and the X-ray pumping rates. When the FUV heating or the UV pumping process dominates, however, the changes will be small. In the following sections we neglect these effects for simplicity.

As the dust particles evolve in the disk, the gas density profile also changes since it is related to the gas temperature profile. In Figure 9 we plot the gas density profiles in the vertical direction at the disk radii of $x=1,10$, and $100 \mathrm{AU}$, which are calculated by using dust model B in quiescent (solid lines) and turbulent (dashed lines) disks. The profiles for the dense cloud dust model are also plotted together as thin solid $(x=1 \mathrm{AU})$, dotted $(x=10 \mathrm{AU})$, and dot-dashed ( $x=100 \mathrm{AU})$ lines for comparison. The gas densities in the models with the dust evolution are lower at the disk midplane and higher at the disk surface than those for the dense cloud dust model because of higher gas temperatures at the midplane and higher disk scale height $\left(H=c_{s 0} / \Omega_{\mathrm{K}}\right)$. In Figure 10 we present the contour plots of the resulting gas temperature (solid lines) and density (dashed lines) profiles in the $z / x$ versus $x$ plane. The contour levels are taken as $T=30,100$, 300,1000 , and $3000 \mathrm{~K}$ and $\rho=10^{-16}, 10^{-14}, 10^{-12}$, and $10^{-10} \mathrm{~g} \mathrm{~cm}^{-3}$. Dust model A with $a_{\max }$ of $(a) 10 \mu \mathrm{m},(b) 1 \mathrm{~mm}$, and $(c) 10 \mathrm{~cm}$ and model B in $(d)$ quiescent and $(e)$ turbulent disks are used in these figures.

Making use of the obtained density and temperature distributions, we calculate the continuum radiation of thermal dust emission from the disks by solving the radiative transfer equation, simply assuming that the disks are face-on to an observer. The resulting IR spectra basically reproduce the median SED observed toward CTTSs in the Taurus-Auriga molecular cloud (D'Alessio et al. 1999, 2006) for dust model A with $a_{\max }=10 \mu \mathrm{m}$. For the models with larger maximum dust radii, the resulting IR dust emission is weaker than the median SED by a factor of about 4 at the most (e.g., D'Alessio et al. 2001). The thermal dust emission from the disks for dust model B in both quiescent and turbulent disks also reproduces the median observed SED if we adjust the inner disk radii. These models do not reproduce the flux deficits relative to the median SED in the NIR to the MIR wavelength bands that are observed toward several CTTSs, including TW Hya (e.g., Calvet et al. 2002; Bergin et al. 2004). However, the disk structure beyond several tens of AU where molecular hydrogen lines are mainly emitted (see Paper I) will be almost unaffected, even if we were to modify the structure of the inner disk within several AU in order to force the thermal dust emission to account for the flux deficits, because only a limited region close to the midplane of the outer disk can be shadowed by the inner disk since the disk has a flared structure.

Finally, in Figure 11 we show another effect of the dust evolution due to the change in grain opacity. The figure shows the profiles of the integrated FUV radiation fields for the energy range of $6 \mathrm{eV}<h \nu<13 \mathrm{eV}$ in the vertical direction at the disk radii of $x=1,10$, and 100 AU. The profiles for dust models A and B are plotted in Figures $11 a$ and $11 b$, respectively, in the same way as Figure 8. The figures show that as the dust particles evolve in the disk and $f_{\text {dust }}$ decreases, the FUV radiation from the central star penetrates deeper into the disk due to the decrease of grain opacity (see also Paper I for the FUV radiation fields in disks).

\subsection{Level Populations of Molecular Hydrogen}

Making use of the physical properties of the disks obtained in the previous subsections, we calculate the level populations of molecular hydrogen in the disks by solving the equations for statistical equilibrium $(\S 2.4)$. The effects of X-ray irradiation and dust evolution on the level populations are discussed in the following.

\subsubsection{Effect of X-Rays}

In Figure 12 we plot the resulting level populations of molecular hydrogen for the models in which the disk is irradiated by (a) both X-ray and UV radiation from the central star, (b) UV radiation only, and $(c) \mathrm{X}$-ray radiation only. Dust model A with maximum dust radius of $a_{\max }=10 \mu \mathrm{m}(\operatorname{see} \S 2.1)$ is used in this subsection. The filled diamonds show the column densities of molecular hydrogen in each rovibrational level as a function of the level energy. The column densities are calculated by integrating the number density of molecular hydrogen in each level along the vertical direction at a disk radius of $50 \mathrm{AU}$. The level populations in local thermodynamic equilibrium (LTE) are shown as dashed lines. The figure shows that if we take into account UV irradiation from the central star, the gas temperature becomes high enough for the collisional excitation process to be very efficient, and the level populations in lower energy levels are in LTE distribution as a result. Meanwhile, if the disk is irradiated by X-rays only and the gas is cold enough, the populations are not in LTE due to the X-ray pumping process. It suggests that we may be able to observe molecular hydrogen transitions excited by X-ray 

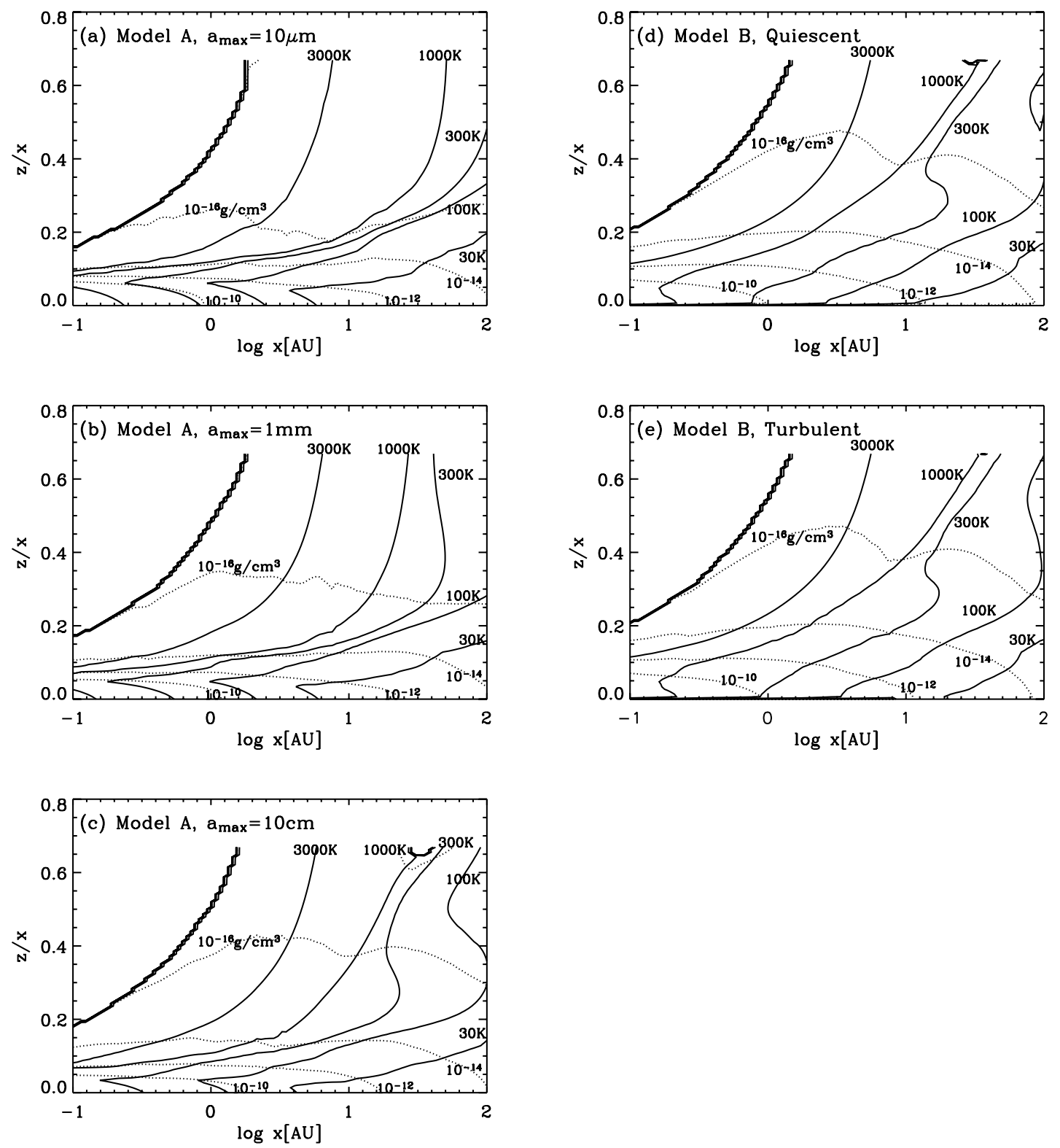

FIg. 10. - Contour plots of the gas temperature (solid lines) and density (dotted lines) distributions in the $z / x$ vs. $x$ plane for dust model A with $a_{\max }$ of $(a) 10 \mu$ m, (b) $1 \mathrm{~mm}$, and (c) $10 \mathrm{~cm}$ and model B in $(d)$ quiescent and (e) turbulent disks. The irradiation model of X-rays $+\mathrm{UV}$ is used here.
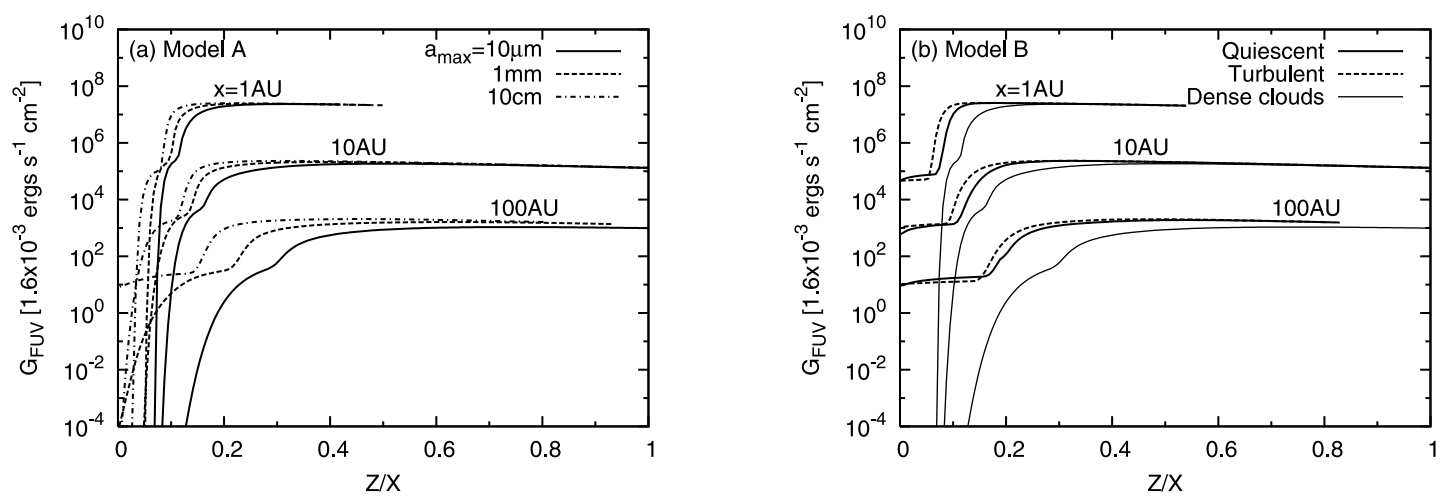

FIG. 11. - Vertical profiles of the integrated FUV radiation fields $(6 \mathrm{eV}<h \nu<13 \mathrm{eV})$ at the disk radii of $x=1,10$, and 100 AU for dust models $(a)$ A and $(b)$ B. The profiles are plotted in the same way as in Fig. 8. The FUV radiation from the central star penetrates deeper into the disks as the dust particles evolve and the grain opacity decreases. 

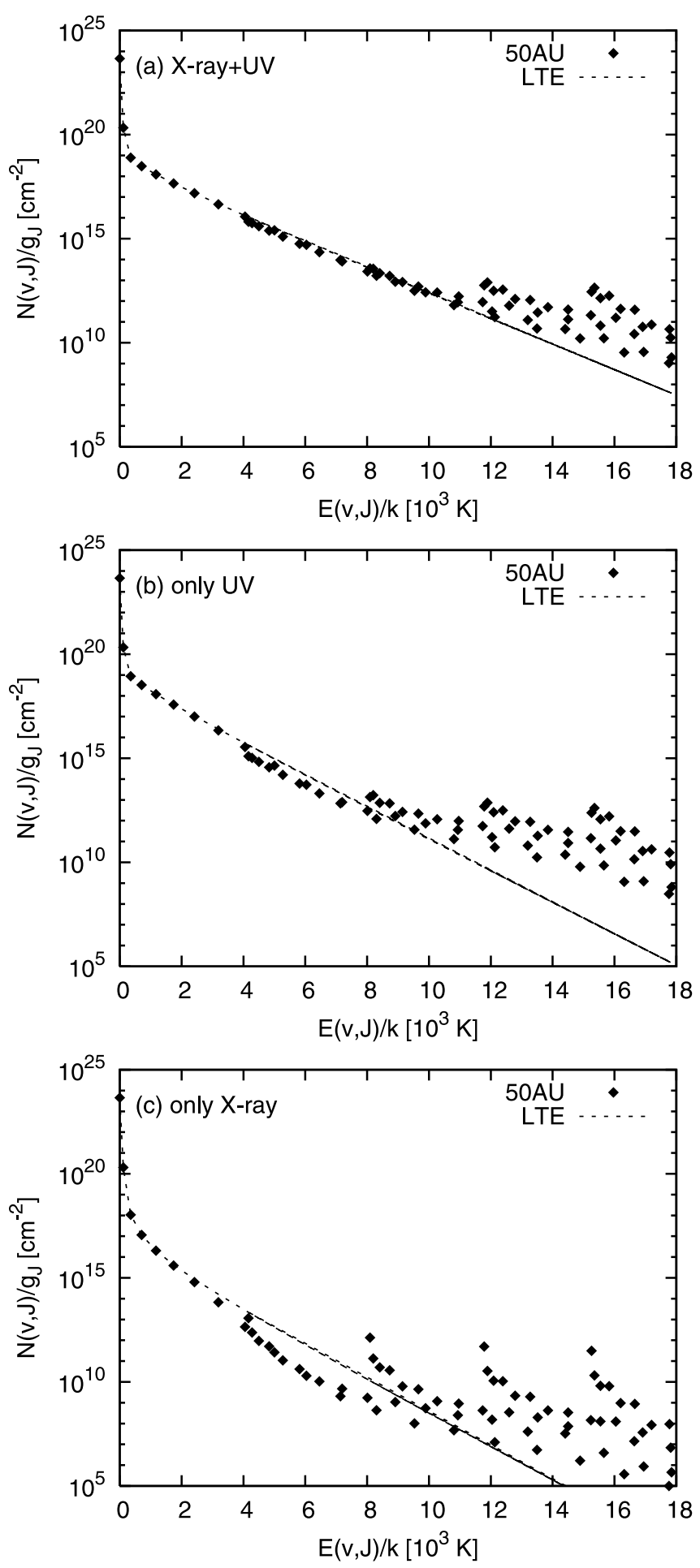

FIG. 12.- - Level populations of molecular hydrogen at a disk radius of $50 \mathrm{AU}$ ( filled diamonds) for the irradiation models of (a) X-rays+UV, (b) UV only, and (c) X-rays only. The LTE distributions are plotted as dashed lines. Dust model A with $a_{\max }=10 \mu \mathrm{m}$ is used here. The populations are in LTE in lower energy levels when the disk is irradiated by strong UV radiation, while they are controlled by $\mathrm{X}$-ray pumping if the $\mathrm{UV}$ irradiation is weak and X-ray irradiation is strong.

pumping toward those protoplanetary disks whose central stars have strong X-ray and weak UV radiation.

\subsubsection{Effect of Dust Evolution}

We now discuss the effect of dust evolution on the level populations of molecular hydrogen in a disk irradiated by both X-ray and UV radiation. Figure 13 is the same as Figure 12, but for different dust models. Dust model A with maximum dust radii $a_{\max }$ of (a) $10 \mu \mathrm{m},(b) 1 \mathrm{~mm}$, and (c) $10 \mathrm{~cm}$ and $(d)$ model B are used in these figures. Figures $13 a-13 c$ show that as the dust particles grow, the level populations of molecular hydrogen change from LTE to non-LTE distributions. This is because with increasing dust size and decreasing $f_{\text {dust }}$, the gas temperature drops due to the decrease of grain photoelectric heating rate, and the collisional excitation process becomes less efficient. In addition, the UV radiation from the central star can penetrate deeper into the disk due to the decrease of grain opacity, and the UV pumping process becomes more efficient. X-ray pumping is not the dominant process if we take into account the UV irradiation from the central star. In Figure $13 d$ the level populations with dust model B in quiescent (asterisks) and turbulent (open squares) disks are plotted together. The populations in quiescent and turbulent disks are almost identical because of similar physical properties of the disks (see $\S 3.2 .2$ ). The figure shows that if we take into account dust evolution, the level populations are in non-LTE distributions in both quiescent and turbulent disks because of low $f_{\text {dust }}$, which results in low gas temperature and high UV radiation fields, while they are in LTE distributions with the dust model of dense clouds (dust model A with $a_{\max }=10 \mu \mathrm{m}$ ).

\subsection{Molecular Hydrogen Emission}

Making use of the physical properties of the disks and the level populations of molecular hydrogen obtained in the previous subsections, we calculate the line emission from molecular hydrogen $(\S 2.4)$. In the following we show the resulting line spectra in the NIR, MIR, and UV wavelength bands and present line ratios, using various dust and irradiation models.

\subsubsection{Line Spectra}

Figures 14, 15, and 16 show the resulting line spectra in the NIR, MIR, and UV wavelength bands, respectively. Dust model A with $a_{\max }$ of (a) $10 \mu \mathrm{m},(b) 1 \mathrm{~mm}$, and (c) $10 \mathrm{~cm}$ and $(d)$ model $\mathrm{B}$ in a quiescent disk are used in these figures. The spectra from a turbulent disk are not plotted in these figures as they are almost identical to those in a quiescent disk.

In Figure 14 and Table 1 (rows with $\lambda<3 \mu \mathrm{m}$ ) we present the rovibrational line fluxes of molecular hydrogen in the NIR wavelength band. These show that as the dust particles grow, the transition lines from higher vibrational energy levels become relatively stronger in this wavelength band. This is because the level populations change from LTE to non-LTE distributions due to the decrease of the gas temperature and the increasing importance of UV pumping, so that the populations in higher vibrational energy levels become relatively larger as we have seen in the previous section (see Fig. 13). The line fluxes decrease with the dust evolution because the area of the high-temperature region in the disk shrinks. In Figure 15 and Table 1 (rows with $\lambda>6 \mu \mathrm{m}$ ) we present the pure rotational transition lines in the MIR wavelength band. In this case the transition lines from lower energy levels become relatively stronger. This is because the level populations in the ground vibrational state are in LTE for all dust models, and the populations in lower energy levels become relatively larger as the gas temperature decreases with increasing dust size. The line fluxes decrease with dust evolution for the same reason as the NIR lines. The MIR flux for dust model B, however, does not decrease so much because $f_{\text {dust }}$ is not very small in the outer disk (see Fig. 2a). The line fluxes from lower energy levels are rather stronger than those in model A since the gas temperatures near the midplane, to which the MIR lines are sensitive, are higher for model B ( $\operatorname{see} \S 3.2 .2$ ). In Table 1 we also list the infrared line 

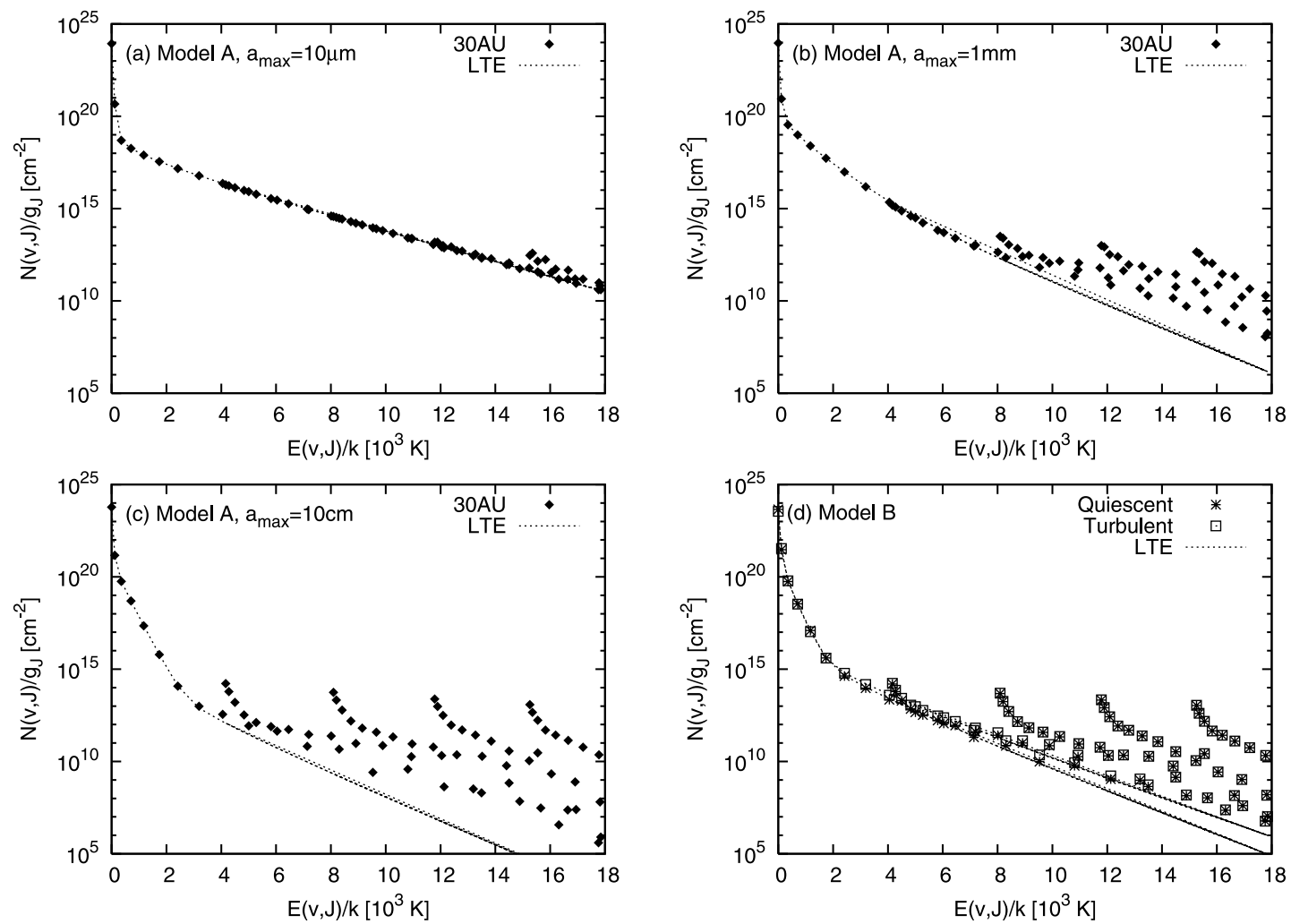

FIG. 13.-Level populations of molecular hydrogen at a disk radius of $30 \mathrm{AU}$ for dust model A ( filled diamonds) with $a_{\max }$ of $(a) 10 \mu \mathrm{m},(b) 1 \mathrm{~mm}$, and $(c) 10 \mathrm{~cm}$ and (d) model B in quiescent (asterisks) and turbulent (open squares) disks. The irradiation model of X-rays+UV is used here. The level populations change from LTE to non-LTE as dust particles grow or settle toward the disk midplane, and since the gas temperature drops while the UV photons in the disk increase.
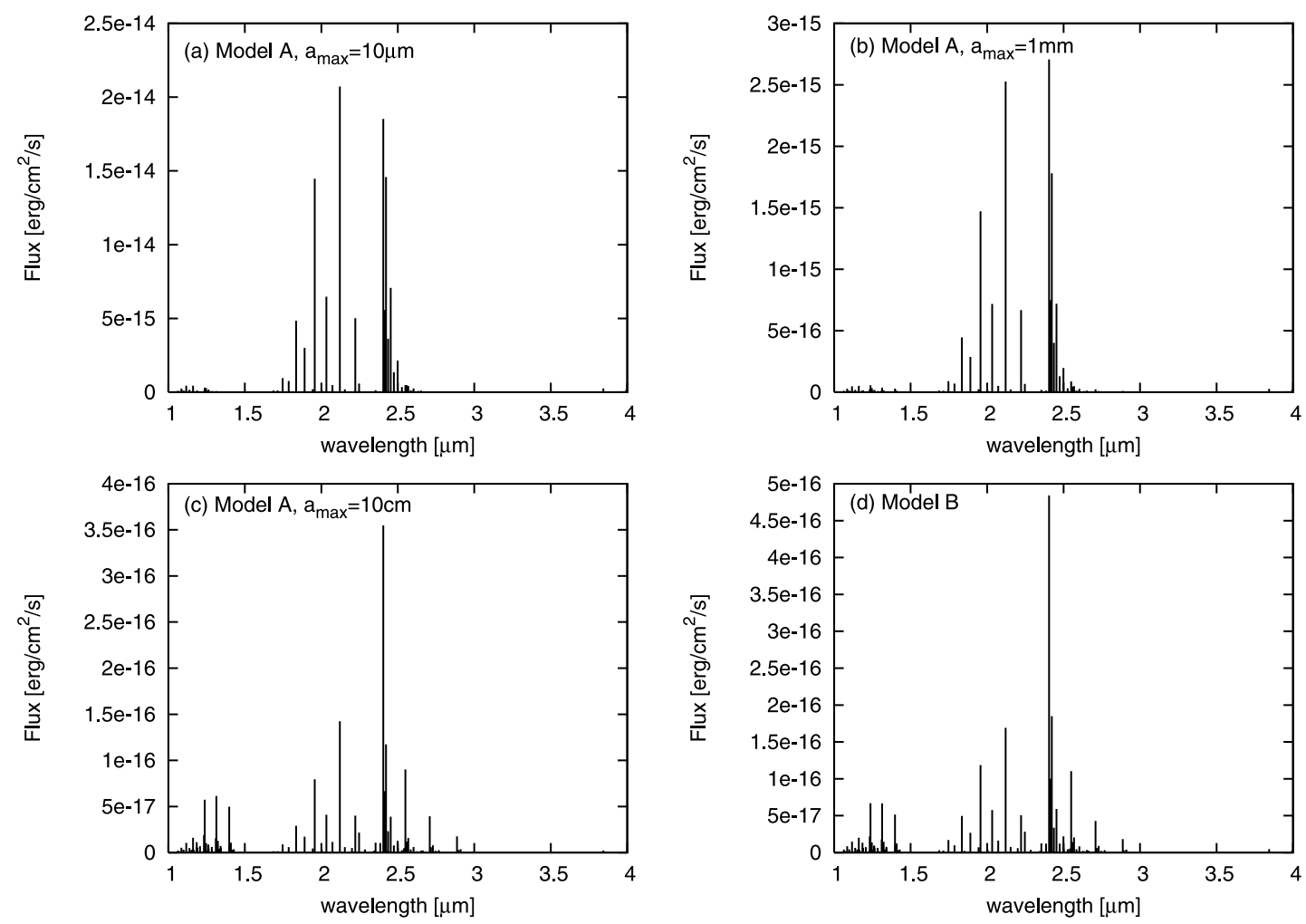

FIG. 14.-NIR $(1 \mu \mathrm{m}<\lambda<4 \mu \mathrm{m})$ spectra of rovibrational transition lines of molecular hydrogen from the disks for dust model A with $a_{\max }$ of $(a) 10 \mu \mathrm{m},(b) 1 \mathrm{~mm}$, and $(c) 10 \mathrm{~cm}$ and $(d)$ model $\mathrm{B}$ in a quiescent disk. The irradiation model of X-rays $+\mathrm{UV}$ is used here. The distance to the disk is set to be $d=56 \mathrm{pc}$. The lines from higher energy levels become relatively stronger as the dust particles evolve and the level populations change from LTE to non-LTE. 

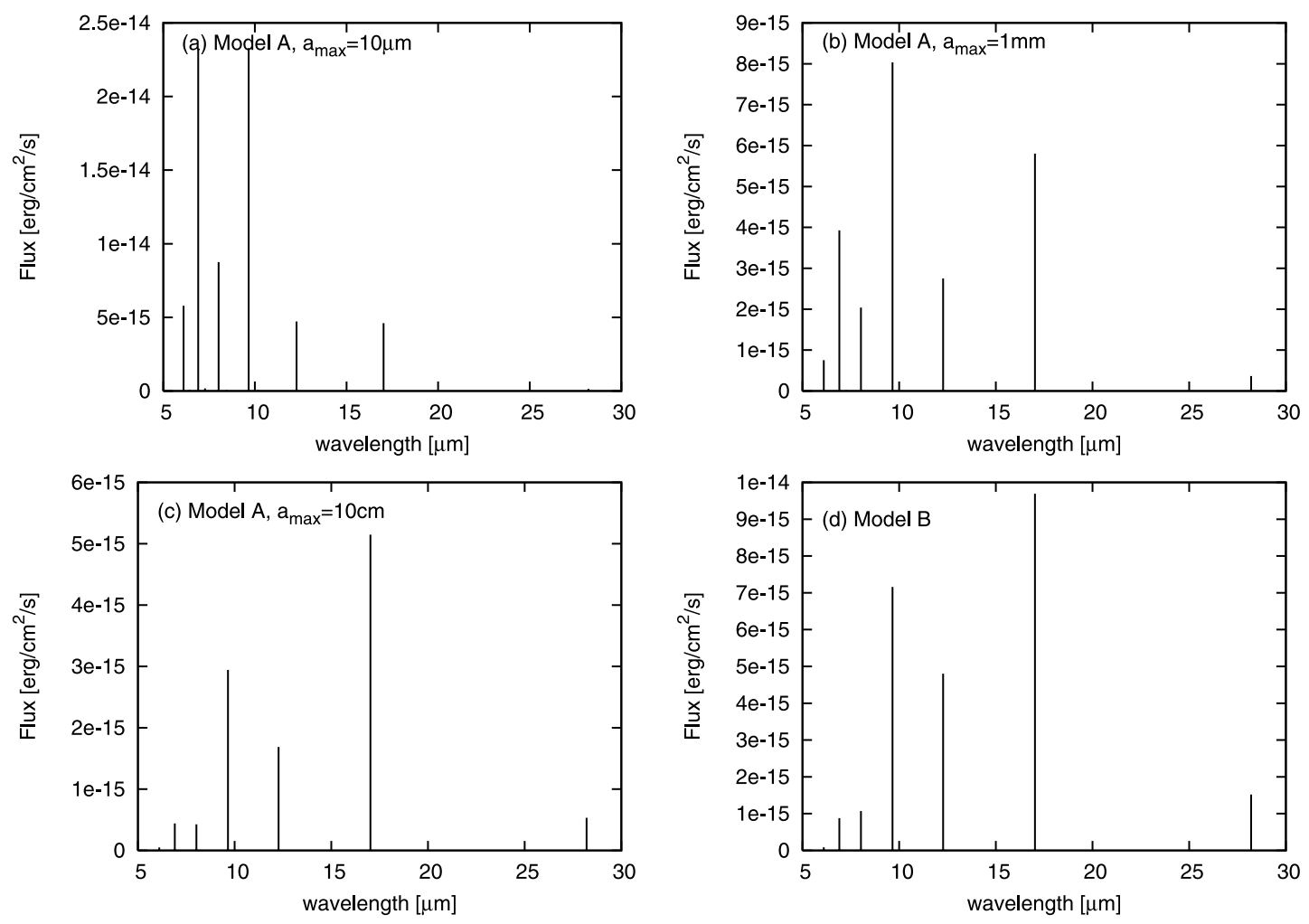

FIG. 15.- Same as Fig. 14, but for the MIR $(5 \mu \mathrm{m}<\lambda<30 \mu \mathrm{m})$ spectra of pure rotational emission lines. The lines from lower energy levels become relatively stronger as dust particles evolve and the gas temperature decreases.
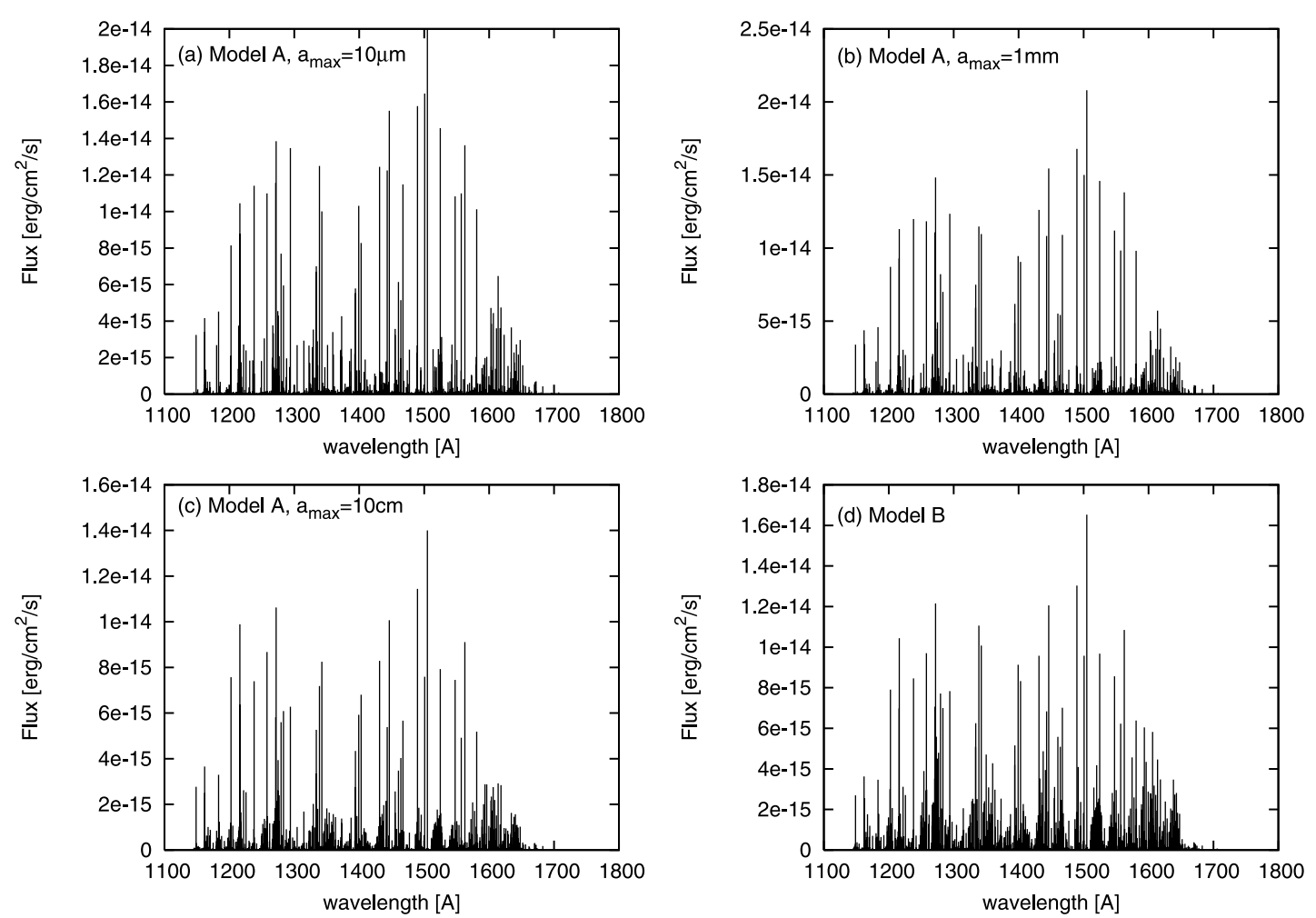

FIg. 16.- Same as Fig. 14, but for the UV (1100 $\AA<\lambda<1800 \AA$ ) emission lines. The line fluxes of transitions originally pumped from higher energy levels seem to be relatively weaker as dust particles evolve and the gas temperature decreases. The lines are also affected by the strength of UV radiation field in the disk. 
TABLE 1

Calculated Infrared Line Fluxes of Molecular Hydrogen

\begin{tabular}{|c|c|c|c|c|c|c|c|c|}
\hline \multirow[b]{3}{*}{$\begin{array}{c}\lambda \\
(\mu \mathrm{m})\end{array}$} & \multirow[b]{3}{*}{ Transition } & \multicolumn{7}{|c|}{$F_{\text {line }}\left(10^{-15} \operatorname{ergs~s}^{-1} \mathrm{~cm}^{-2}\right)$} \\
\hline & & \multicolumn{5}{|c|}{ Model A $\left(a_{\max }\right)$} & \multicolumn{2}{|c|}{ Model B } \\
\hline & & $\begin{array}{l}\text { X-Rays } \\
10 \mu \mathrm{m}\end{array}$ & $\begin{array}{c}\mathrm{UV} \\
10 \mu \mathrm{m}\end{array}$ & $\begin{array}{c}\text { X-Rays+UV } \\
10 \mu \mathrm{m}\end{array}$ & $\begin{array}{c}\text { X-Rays+UV } \\
1 \mathrm{~mm}\end{array}$ & $\begin{array}{c}\text { X-Rays+UV } \\
10 \mathrm{~cm}\end{array}$ & $\begin{array}{c}\text { X-Rays+UV } \\
\text { Quiescent }\end{array}$ & $\begin{array}{c}\text { X-Rays+UV } \\
\text { Turbulent }\end{array}$ \\
\hline $1.16 \ldots \ldots$ & $2-0 S(1)$ & 0.068 & 0.182 & 0.439 & 0.051 & 0.016 & 0.020 & 0.019 \\
\hline $1.19 \ldots \ldots \ldots \ldots \ldots \ldots$ & $2-0 S(0)$ & 0.013 & 0.039 & 0.091 & 0.013 & 0.005 & 0.006 & 0.006 \\
\hline $1.24 \ldots \ldots \ldots \ldots \ldots \ldots$ & $2-0 Q(1)$ & 0.043 & 0.143 & 0.310 & 0.056 & 0.057 & 0.067 & 0.061 \\
\hline $1.24 \ldots \ldots \ldots \ldots \ldots \ldots$ & $2-0 Q(2)$ & 0.014 & 0.041 & 0.095 & 0.014 & 0.008 & 0.009 & 0.009 \\
\hline $1.25 \ldots \ldots \ldots \ldots \ldots \ldots$ & $2-0 Q(3)$ & 0.043 & 0.116 & 0.280 & 0.033 & 0.010 & 0.014 & 0.013 \\
\hline $1.83 \ldots .$. & $1-0 S(5)$ & 0.587 & 2.286 & 4.849 & 0.446 & 0.029 & 0.050 & 0.049 \\
\hline $1.89 \ldots \ldots \ldots \ldots \ldots \ldots$ & $1-0 S(4)$ & 0.314 & 1.555 & 3.007 & 0.287 & 0.017 & 0.027 & 0.026 \\
\hline $1.96 \ldots \ldots \ldots \ldots \ldots \ldots$ & $1-0 S(3)$ & 1.326 & 8.096 & 14.475 & 1.472 & 0.079 & 0.119 & 0.111 \\
\hline $2.03 \ldots \ldots \ldots \ldots \ldots \ldots$ & $1-0 S(2)$ & 0.533 & 3.857 & 6.477 & 0.719 & 0.041 & 0.058 & 0.051 \\
\hline $2.12 \ldots \ldots \ldots \ldots \ldots$ & $1-0 S(1)^{\mathrm{a}}$ & 1.565 & 12.968 & 20.725 & 2.526 & 0.142 & 0.169 & 0.135 \\
\hline $2.22 \ldots \ldots \ldots \ldots \ldots \ldots$ & $1-0 S(0)$ & 0.357 & 3.256 & 5.023 & 0.667 & 0.040 & 0.051 & 0.037 \\
\hline $2.25 \ldots \ldots \ldots \ldots \ldots \ldots$ & $2-1 S(1)$ & 0.090 & 0.242 & 0.583 & 0.068 & 0.022 & 0.028 & 0.026 \\
\hline $2.35 \ldots \ldots \ldots \ldots \ldots \ldots$ & $2-1 S(0)$ & 0.019 & 0.056 & 0.130 & 0.019 & 0.011 & 0.013 & 0.012 \\
\hline $2.41 \ldots \ldots \ldots \ldots \ldots \ldots$ & $1-0 Q(1)$ & 1.270 & 12.299 & 18.528 & 2.707 & 0.355 & 0.484 & 0.373 \\
\hline $2.41 \ldots \ldots \ldots \ldots \ldots . .$. & $1-0 Q(2)$ & 0.395 & 3.604 & 5.560 & 0.748 & 0.066 & 0.100 & 0.077 \\
\hline $2.42 \ldots \ldots \ldots \ldots \ldots \ldots$ & $1-0 Q(3)$ & 1.101 & 9.128 & 14.588 & 1.780 & 0.117 & 0.185 & 0.145 \\
\hline $2.44 \ldots \ldots \ldots \ldots \ldots \ldots$ & $1-0 Q(4)$ & 0.298 & 2.156 & 3.621 & 0.402 & 0.023 & 0.034 & 0.029 \\
\hline $2.45 \ldots \ldots \ldots \ldots \ldots \ldots$ & $1-0 Q(5)$ & 0.648 & 3.954 & 7.069 & 0.719 & 0.039 & 0.059 & 0.055 \\
\hline $2.47 \ldots \ldots \ldots \ldots \ldots \ldots$ & $1-0 Q(6)$ & 0.142 & 0.702 & 1.357 & 0.129 & 0.008 & 0.012 & 0.012 \\
\hline $2.50 \ldots \ldots \ldots \ldots \ldots \ldots$ & $1-0 Q(7)$ & 0.260 & 1.011 & 2.145 & 0.197 & 0.013 & 0.022 & 0.021 \\
\hline $2.55 \ldots \ldots \ldots \ldots \ldots \ldots$ & $2-1 Q(1)$ & 0.066 & 0.223 & 0.483 & 0.088 & 0.090 & 0.110 & 0.097 \\
\hline $2.56 \ldots \ldots \ldots \ldots \ldots \ldots$ & $2-1 Q(2)$ & 0.022 & 0.063 & 0.147 & 0.021 & 0.012 & 0.014 & 0.013 \\
\hline $2.57 \ldots \ldots \ldots \ldots \ldots \ldots$ & $2-1 Q(3)$ & 0.066 & 0.176 & 0.424 & 0.050 & 0.016 & 0.021 & 0.019 \\
\hline $6.11 \ldots \ldots \ldots \ldots \ldots \ldots$ & $0-0 S(6)$ & 0.368 & 3.949 & 5.791 & 0.755 & 0.050 & 0.084 & 0.058 \\
\hline 6.91_................ & $0-0 S(5)$ & 1.203 & 17.346 & 23.219 & 3.928 & 0.441 & 0.880 & 0.564 \\
\hline $8.02 \ldots \ldots \ldots \ldots \ldots \ldots$ & $0-0 S(4)$ & 0.375 & 7.023 & 8.753 & 2.038 & 0.427 & 1.072 & 0.731 \\
\hline $9.66 \ldots \ldots \ldots \ldots \ldots \ldots$ & $0-0 S(3)$ & 0.849 & 19.835 & 23.286 & 8.035 & 2.943 & 7.160 & 6.140 \\
\hline $12.27 \ldots \ldots \ldots \ldots \ldots$ & $0-0 S(2)^{\mathrm{b}}$ & 0.163 & 4.271 & 4.719 & 2.751 & 1.688 & 4.803 & 4.711 \\
\hline 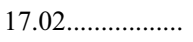 & $0-0 S(1)^{\mathrm{b}, \mathrm{c}}$ & 0.209 & 4.466 & 4.606 & 5.806 & 5.148 & 9.690 & 9.900 \\
\hline $28.20 \ldots \ldots \ldots \ldots \ldots$ & $0-0 S(0)^{\mathrm{c}}$ & 0.020 & 0.145 & 0.142 & 0.365 & 0.532 & 1.521 & 1.717 \\
\hline
\end{tabular}

${ }^{a}$ Ground-based observations by Bary et al. (2003) and Itoh et al. (2003) give fluxes of $(1.0-15) \times 10^{-15} \mathrm{ergs} \mathrm{s}^{-1} \mathrm{~cm}^{-2}$.

${ }^{\mathrm{b}}$ Ground-based observations by Richter et al. (2002) and Sako et al. (2005) give fluxes of $<30 \times 10^{-15} \mathrm{ergs} \mathrm{s}^{-1} \mathrm{~cm}^{-2}$ for the $12 \mu \mathrm{m} \mathrm{S}(2) \operatorname{line}$ and $<39 \times 10^{-15}$ ergs s${ }^{-1}$ $\mathrm{cm}^{-2}$ for the $17 \mu \mathrm{m} S(1)$ line.

${ }^{c}$ Infrared Space Observatory observations by Thi et al. (2001a) give fluxes of (28-81) $\times 10^{-15} \mathrm{ergs} \mathrm{s}^{-1} \mathrm{~cm}^{-2}$ for the $17 \mu \mathrm{m} \mathrm{S}(1) \operatorname{line}$ and $(25-57) \times 10^{-15} \mathrm{ergs} \mathrm{s}^{-1} \mathrm{~cm}^{-2}$ for the $28 \mu \mathrm{m} S(0)$ line.

fluxes for different irradiation models, calculated by using dust model A with $a_{\max }=10 \mu \mathrm{m}$. For the irradiation model of X-rays only, the intensity of NIR lines is similar to that for the irradiation model of X-rays+UV and dust model A with $a_{\max }=1 \mathrm{~mm}$. The MIR lines are relatively weaker because the gas temperature at the outer disk is not so high if the irradiation source is X-rays only (see Fig. 5). For the irradiation model of UV only, the NIR and MIR line fluxes are a bit weaker than those for the irradiation model of $\mathrm{X}$-rays $+\mathrm{UV}$ and dust model A with $a_{\max }=10 \mu \mathrm{m}$ since the gas temperature for the latter model is higher due to the X-ray heating. The intensity of emission lines from lower energy levels in the MIR is similar between the former and the latter models because $\mathrm{X}$-ray heating does not affect the gas temperature in the outer disk.

Comparing our results for the $2.12 \mu \mathrm{m} v=1 \rightarrow 0 S(1)$ transition to the observational data toward TW Hya of $1.0 \times$ $10^{-15} \mathrm{ergs} \mathrm{s}^{-1} \mathrm{~cm}^{-2}$ (Bary et al. 2003), dust model A with $a_{\max }=$ $1 \mathrm{~mm}\left(f_{\text {dust }}=0.1\right)$ seems to be most suitable. We may need a larger amount of small dust grains than that predicted in dust model B in order to reproduce the observed $2.12 \mu \mathrm{m}$ line flux. The calculated fluxes of the MIR lines are consistent with the upper limits of the ground-based observations (Richter et al. 2002; Sako et al. 2005).
In Figure 16 and Table 2 we present the line fluxes in the UV wavelength band. In the table we list the lines pumped by $0-2$ $R(0), 0-2 R(1), 1-2 P(5), 1-2 R(6), 3-1 P(14)$, and 4-3 $P(5)$ transitions in the wavelength band of the strong Ly $\alpha$ emission from the central star (see, e.g., Herczeg et al. 2002; Paper I). The figure and table show that the fluxes of most lines decrease as dust particles evolve in the disk and the area of the high-temperature region shrinks. The fluxes of some lines, however, do not decrease because the lines in the UV wavelength band, excited by UV photons, are affected very much by the strength of the UV radiation field in the disk, which becomes stronger with dust evolution (see Fig. 11). The line fluxes for dust model B are relatively strong since the populations of molecular hydrogen in the energy levels in the ground electronic states, from which the lines are pumped, are larger. The populations in lower energy levels are large because the gas temperatures near the midplane are high, while those in higher energy levels, which are controlled by the UV pumping process, are large because the UV irradiation from the central star penetrates deeper into the disk (see Figs. 8 and 11). We note that in this work the fluxes of lines in the UV wavelength band caused by $\mathrm{X}$-ray pumping are not calculated. Such pumping will affect the strength of weak emission lines of molecular hydrogen (e.g., 
TABLE 2

Calculated and Observed Ultraviolet Line Fluxes of Molecular Hydrogen

\begin{tabular}{|c|c|c|c|c|c|c|c|c|c|c|c|c|c|c|c|}
\hline \multirow{2}{*}{$\begin{array}{c}\lambda \\
(\AA)\end{array}$} & \multirow{2}{*}{ Transition } & \multicolumn{6}{|c|}{$F_{\text {line }}\left(10^{-15} \mathrm{ergs} \mathrm{s}^{-1} \mathrm{~cm}^{-2}\right)$} & \multirow{2}{*}{$\begin{array}{c}\lambda \\
(\AA)\end{array}$} & \multirow{2}{*}{ Transition } & \multicolumn{6}{|c|}{$F_{\text {line }}\left(10^{-15} \mathrm{ergs} \mathrm{s}^{-1} \mathrm{~cm}^{-2}\right)$} \\
\hline & & \multicolumn{3}{|c|}{ Model A $\left(a_{\max }\right)$} & \multicolumn{2}{|c|}{ Model B } & Oвs. ${ }^{\mathrm{a}}$ & & & \multicolumn{3}{|c|}{ Model A $\left(a_{\max }\right)$} & \multicolumn{2}{|c|}{ Model B } & \\
\hline & & Pump & ped by $c$ & $-2 R(0)$ & & & & & & & Imped by & $0-2 R(1)$ & & & \\
\hline $1161.7 \ldots \ldots$. & $0-1 R(0)$ & 0.436 & 0.427 & 0.291 & 0.311 & 0.300 & & $1162.2 \ldots \ldots$ & $0-1 R(1)$ & 0.501 & 0.512 & 0.412 & 0.428 & 0.424 & \\
\hline $1222.0 \ldots \ldots$ & $0-2 P(2)$ & 2.719 & 3.039 & 2.623 & & & & $1225.6 \ldots \ldots$ & $0-2 P(3)$ & 2.395 & 2.683 & 2.538 & 2.715 & 2.789 & \\
\hline $1274.6 \ldots \ldots$ & $0-3 R(0)$ & 4.550 & 4.478 & 2.608 & 3.630 & 3.954 & 27.4 & $1275.0 \ldots \ldots$ & $0-3 R(1)$ & 4.304 & 4.918 & 3.941 & 4.506 & 4.624 & 24.6 \\
\hline $1279.6 \ldots \ldots$ & $0-3 P(2)$ & 7.690 & 8.210 & 5.606 & 7.717 & 8.722 & 39.2 & $1283.2 \ldots \ldots$ & $0-3 P(3)$ & 5.950 & 6.996 & 6.092 & 7.000 & 7.443 & 28.0 \\
\hline $1333.6 \ldots \ldots$ & $0-4 R(0)$ & 6.694 & 5.909 & 3.344 & 5.087 & 5.683 & 42.8 & $1333.9 \ldots \ldots$ & $0-4 R(1)$ & 7.005 & 7.487 & 5.268 & 6.252 & 6.606 & 7.9 \\
\hline $1338.7 \ldots \ldots$. & $0-4 P(2)$ & 12.495 & 11.472 & 7.185 & 11.063 & 12.584 & 73.1 & $1342.4 \ldots \ldots$ & $0-4 P(3)$ & 10.007 & 10.958 & 8.251 & 10.080 & 11.168 & 64.9 \\
\hline $1393.9 \ldots \ldots$ & $0-5 R(0)$ & 5.516 & 4.866 & 2.757 & 4.199 & 4.693 & 35.3 & $1394.1 \ldots \ldots$ & $0-5 R(1)$ & 5.790 & 6.177 & 4.344 & 5.159 & 5.456 & 52.4 \\
\hline $1577.3 \ldots \ldots$ & $0-8 R(0)$ & 0.302 & 0.258 & 0.164 & 0.291 & 0.315 & & $1577.1 \ldots \ldots$ & $0-8 R(1)$ & 0.378 & 0.361 & 0.259 & 0.362 & 0.412 & $\ldots$ \\
\hline $1582.6 \ldots \ldots$ & $0-8 P(2)$ & 0.592 & 0.509 & 0.330 & 0.593 & 0.637 & & $1586.0 \ldots \ldots$ & $0-8 P(3)$ & 0.553 & 0.531 & 0.389 & 0.590 & 0.662 & \\
\hline $1636.4 \ldots \ldots$ & $0-9 R(0)$ & 0.034 & 0.029 & 0.019 & 0.035 & 0.037 & & $1636.1 \ldots \ldots$ & $0-9 R(1)$ & 0.044 & 0.041 & 0.030 & 0.048 & 0.053 & \\
\hline $1641.7 \ldots \ldots$ & $0-9 P(2)$ & 0.067 & 0.058 & 0.038 & 0.069 & 0.073 & & $1644.8 \ldots \ldots$ & $0-9 P(3)$ & 0.064 & 0.061 & 0.044 & .073 & 0.079 & \\
\hline $1692.5 \ldots \ldots$ & $0-10 R(0)$ & 0.001 & 0.001 & 0.000 & 0.001 & 0.001 & & $1692.0 \ldots \ldots$ & $0-10 R(1)$ & 0.001 & 0.001 & 0.001 & 0.001 & 0.001 & \\
\hline $1697.6 \ldots \ldots$ & $0-10 P(2)$ & 0.002 & 0.001 & 0.001 & 0.002 & 0.002 & & $1700.3 \ldots \ldots$ & $0-10 P(3)$ & 0.002 & 0.001 & 0.001 & 0.002 & 0.002 & \\
\hline & & Pump & ped by 1 & $-2 P(5)$ & & & & & & & Imped by & $2 R(6)$ & & & \\
\hline $1148.7 \ldots \ldots$ & $1-1 R(3)$ & 3.247 & 3.386 & 2.774 & 2.701 & 2.688 & 4.6 & $162.0 \ldots \ldots$ & $1-1$ & 3.417 & 3.415 & 2.505 & 2.559 & 2.525 & \\
\hline $1161.9 \ldots \ldots$ & $1-1 P(5)$ & 4.165 & 4.369 & 3.660 & 3.631 & 3.611 & 10.9 & $183.4 \ldots \ldots$ & $1-1 P(8)$ & 4.524 & 4.591 & 3.294 & 67 & 4 & \\
\hline $1202.5 \ldots \ldots$ & $1-2 R(3)$ & 8.145 & 8.716 & 7.575 & 7.909 & 7.881 & 11.3 & $1215.8 \ldots \ldots$ & $1-2 R(6)$ & 8.795 & 9.284 & 6.379 & .986 & .787 & \\
\hline $1216.2 \ldots \ldots$ & $1-2 P(5)$ & 10.449 & 11.295 & 9.889 & 10.442 & 10.425 & & $1238.0 \ldots \ldots$ & $1-2 P(8)$ & 11.411 & 11.984 & 7.394 & .460 & 162 & 11.5 \\
\hline $1489.8 \ldots \ldots$ & $1-7 R(3)$ & 15.769 & 16.791 & 11.439 & 13.034 & 12.946 & 48.2 & $1500.7 \ldots \ldots$ & $1-7 R(6)$ & 16.461 & 15.004 & 7.589 & 9.577 & 9.034 & 19.7 \\
\hline $5.0 \ldots \ldots$ & $1-7 P(5)$ & 19.978 & 20.803 & 14.002 & 16.528 & 16.532 & 57.5 & $1524.9 \ldots \ldots$ & $1-7 P(8)$ & 14.565 & 14.593 & 7.929 & 9.677 & 9.255 & 23.5 \\
\hline $1547.6 \ldots \ldots$ & $1-8 R(3)$ & 10.831 & 11.200 & 7.458 & 8.565 & 8.528 & 35.3 & $1557.2 \ldots \ldots$ & $1-8 R(6)$ & 10.993 & 9.828 & 4.920 & 6.230 & 5.864 & 17.0 \\
\hline $1562.7 \ldots \ldots$ & $1-8 P(5)$ & 13.631 & 13.810 & 9.112 & 10.847 & 10.845 & 37.2 & $1581.0 \ldots \ldots$ & $1-8 P(8)$ & 10.123 & 9.813 & 5.191 & 6.384 & 6.088 & 17.5 \\
\hline $1603.5 \ldots \ldots$ & $1-9 R(3)$ & 3.851 & 3.685 & 2.342 & 2.774 & 2.773 & 11.2 & $1611.3 \ldots \ldots$ & $1-9 R(6)$ & 3.608 & 3.098 & 1.520 & 1.941 & 1.816 & $\ldots$ \\
\hline $1618.2 \ldots \ldots$ & $1-9 P(5)$ & 4.742 & 4.487 & 2.843 & 3.482 & 3.459 & 11.6 & $1634.2 \ldots \ldots$ & $1-9 P(8)$ & 3.653 & 3.256 & 1.630 & 2.048 & 1.935 & 5.5 \\
\hline $1656.2 \ldots \ldots$ & $1-10 R(3)$ & 0.443 & 0.402 & 0.250 & 0.312 & 0.307 & $\ldots$ & $1661.5 \ldots \ldots$ & $1-10 R(6)$ & 0.395 & 0.332 & 0.161 & 0.207 & 0.193 &. \\
\hline $1670.0 \ldots \ldots$ & $1-10 P(5)$ & 0.537 & 0.487 & 0.303 & 0.382 & 0.374 & & $1682.8 \ldots \ldots$ & $1-10 P(8)$ & 0.425 & 0.359 & 0.175 & 0.224 & 0.209 & \\
\hline & & Pum & sed by 3 & (- & & & & & & & 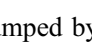 & & & & \\
\hline $1180.3 \ldots \ldots$ & $3-1 R(12)$ & 2.681 & 2.242 & 0.848 & 27 & 777 & . & $.3 \ldots \ldots$ & $4-2 R(3)$ & 0.262 & 0.196 & 0.096 & 153 & 170 & \\
\hline $1214.2 \ldots \ldots$ & $3-1 P(14)$ & 3.749 & 2.662 & 0.854 & 0.919 & 0.846 & $\ldots$ & $1163.8 \ldots \ldots$ & $4-2 P(5)$ & 0.333 & 0.244 & 0.117 & 0.202 & 0.217 & \\
\hline $1231.3 \ldots \ldots$ & $3-2 R(12)$ & 1.839 & 1.133 & 0.338 & 0.405 & 0.354 & $\ldots$ & $1202.0 \ldots \ldots$ & $4-3 R(3)$ & 2.094 & 1.680 & 0.823 & 1.218 & 1.354 & \\
\hline $1266.1 \ldots \ldots$ & $3-2 P(14)$ & 1.651 & 1.064 & 0.324 & 0.367 & 0.331 & & $1214.9 \ldots \ldots$ & $4-3 P(5)$ & 2.699 & 2.108 & 1.025 & 1.666 & 1.815 & . \\
\hline $1283.1 \ldots \ldots$ & $3-3 R(12)$ & 0.345 & 0.203 & 0.059 & 0.075 & 0.063 & & $1253.8 \ldots \ldots$ & $4-4 R(3)$ & 3.058 & 2.094 & 0.945 & 1.597 & 1.745 & 6.0 \\
\hline $1318.7 \ldots \ldots$ & $3-3 P(14)$ & 0.328 & 0.195 & 0.057 & 0.071 & 0.061 & $\ldots$ & $1267.0 \ldots \ldots$ & $4-4 P(5)$ & 3.771 & 2.551 & 1.161 & 2.238 & 2.324 & 12.9 \\
\hline $1335.4 \ldots \ldots$ & $3-4 R(12)$ & 2.896 & 1.914 & 0.590 & 0.671 & 0.605 & $\ldots$ & $1306.4 \ldots \ldots$ & $4-5 R(3)$ & 0.142 & 0.091 & 0.041 & 0.086 & 0.085 & \\
\hline $1371.5 \ldots \ldots$ & $3-4 P(14)$ & 2.432 & 1.746 & 0.564 & 0.605 & 0.557 & 4.0 & $1319.8 \ldots \ldots$ & $4-5 P(5)$ & 0.171 & 0.109 & 0.049 & 0.105 & 0.102 & \\
\hline $1387.7 \ldots \ldots$ & $3-5 R(12)$ & 1.246 & 0.772 & 0.231 & 0.275 & 0.241 & 3.4 & $1359.3 \ldots$ & $4-6 R(3)$ & 3.399 & 2.430 & 1.105 & 1.751 & 1.938 & 6.5 \\
\hline $1423.9 \ldots \ldots$ & $3-5 P(14)$ & 1.121 & 0.729 & 0.2 & & & 1.6 & $1372.9 \ldots \ldots$ & $4-6 P(5)$ & 4.266 & 2.993 & 380 & 2.534 & 2.689 & 7.2 \\
\hline $1439.4 \ldots \ldots$ & $3-6 R(12)$ & 0.597 & 0.360 & 0.106 & & & & $1412.0 \ldots \ldots$ & $4-7 R(3)$ & 0.802 & 0.526 & 0.236 & 0.443 & 0.466 & \\
\hline $1475.2 \ldots \ldots$ & $3-6 P(14)$ & 0.557 & 0.346 & & & & & $1425.6 \ldots \ldots$ & $4-7 P(5)$ & 0.976 & 0.637 & 0.287 & 0.587 & 0.590 & \\
\hline $1489.4 \ldots \ldots$ & $3-7 R(12)$ & 2.669 & 1.871 & 0.595 & & 0.600 & 6.7 & $1463.8 \ldots \ldots$ & $4-8 R(3)$ & 1.957 & 1.360 & 0.615 & 1.014 & 1.115 & 2.3 \\
\hline $1524.3 \ldots \ldots$ & $3-7 P(14)$ & 2.166 & 1.680 & 0.573 & 0.599 & 0.555 & 5.1 & $1477.3 \ldots \ldots$ & $4-8 P(5)$ & 2.440 & 1.671 & 0.764 & 1.446 & 1.515 & 5.8 \\
\hline $1536.8 \ldots \ldots$ & $3-8 R(12)$ & 0.658 & 0.403 & 0.120 & 0.144 & 0.126 & & $\mid 1513.7 \ldots \ldots$. & $4-9 R(3)$ & 2.463 & 1.787 & 0.815 & 1.269 & 1.408 & 4.3 \\
\hline
\end{tabular}


TABLE 2-Continued

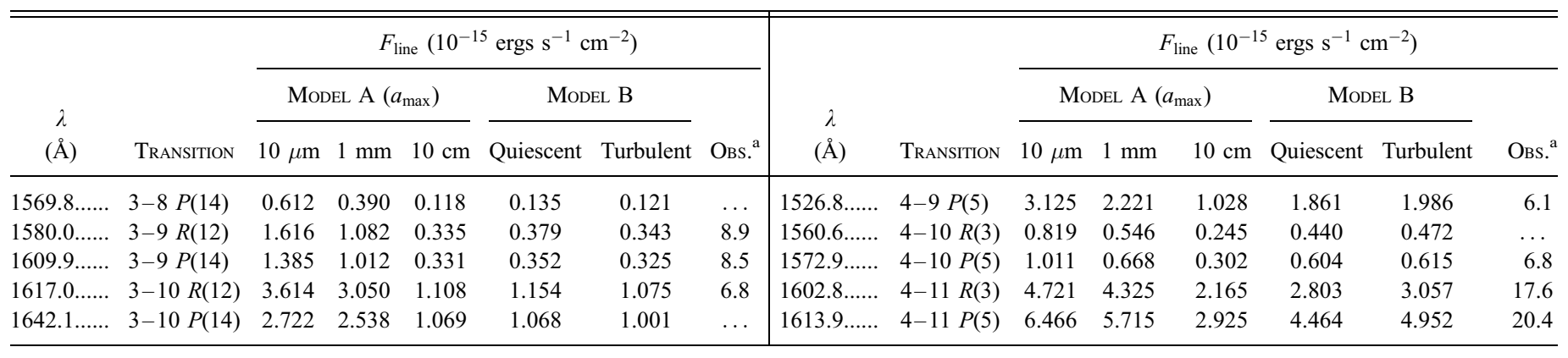

${ }^{\text {a }}$ FUSE and HST observations by Herczeg et al. (2002).

Bergin et al. 2004), but the effect on the flux of the strong lines, listed in Table 2, which are pumped by the strong Ly $\alpha$ line emission, will be negligible.

The calculated UV line fluxes are $10^{-15}$ to $10^{-14} \mathrm{ergs} \mathrm{s}^{-1} \mathrm{~cm}^{-2}$, which are consistent with the observations toward TW Hya (Herczeg et al. 2002) to zeroth order but have discrepancies in details. This could be because the UV line fluxes depend not only on the density and temperature profiles of protoplanetary disks but also on the strength and shape of the Ly $\alpha$ line irradiated from the central star, as shown by Herczeg et al. (2002, 2004; see also Paper I). A simple single Gaussian profile is used for the Ly $\alpha$ line profile in this paper, while the actual line profile seems to be influenced by wind absorption (see Herczeg et al. 2002, 2004). Thus, a more detailed analysis of the Ly $\alpha$ line profile, beyond the scope of this work, will be needed in order to fit the observed UV line fluxes in more detail.

Our results show that molecular hydrogen emission is strong and will be easier to observe toward those disks whose central stars have strong UV and X-ray radiation. In addition, if the disk contains a relatively large amount of small dust grains, the volume of hot gas in the disk will be larger, and emission lines will be stronger. Therefore, the disks that have an observable signature of the presence of small dust grains, such as strong $10 \mu \mathrm{m}$ silicate emission, could be good targets in which to observe molecular hydrogen lines.

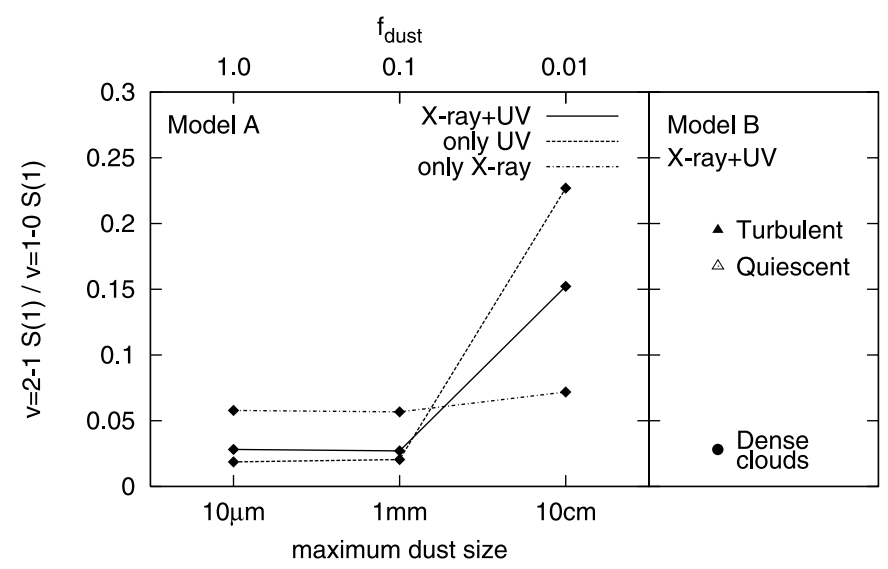

FIG. 17.-The $v=1 \rightarrow 0 S(1) / v=2 \rightarrow 1 S(1)$ line ratios for the irradiation models of X-rays $+\mathrm{UV}$ (solid lines), UV only (dashed lines), and X-rays only (dotdashed lines $)$ and dust model A with $a_{\max }=10 \mu \mathrm{m}, 1 \mathrm{~mm}$, and $10 \mathrm{~cm}\left(f_{\text {dust }}=1.0\right.$, 0.1 , and 0.01 ) (left). The ratios for the irradiation model by X-rays+UV with dust model B in quiescent (open triangle) and turbulent ( filled triangle) disks and the dense clouds (dust model A with $a_{\max }=10 \mu \mathrm{m}$; filled circle) are plotted in the right panel. If the disk is irradiated by strong UV radiation, the line ratio increases with dust evolution as a consequence of the decrease in gas temperature and the increase in the UV radiation field strength.

$$
\text { 3.4.2. } v=1 \rightarrow 0 S(1) / v=2 \rightarrow 1 S(1) \text { Line Ratio }
$$

Finally, we discuss the effect of the dust evolution on a particular line ratio, the $v=1 \rightarrow 0 S(1) / v=2 \rightarrow 1 S(1)$ ratio, which is often used as a probe of physical properties of astronomical objects. In Figure 17 the resulting line ratios for various irradiation models and dust models are plotted. The lines with diamonds in the left panel show the ratios calculated using dust model A with $a_{\max }=10 \mu \mathrm{m}, 1 \mathrm{~mm}$, and $10 \mathrm{~cm}\left(f_{\text {dust }}=1.0,0.1\right.$, and 0.01$)$ and irradiation of both X-rays and UV (solid lines), UV only (dashed lines), and X-rays only (dot-dashed lines). They show that if the disk is irradiated by the UV radiation from the central star, this ratio becomes larger as the dust particles grow and the total surface area of dust grains, $f_{\text {dust }}$, decreases. This is because the level populations of molecular hydrogen change from LTE to non-LTE distributions due to the increase in the grain photoelectric heating rate and the decrease of grain opacity, as discussed in $\S$ 3.3.2. This effect appears to be more efficient if the disk is irradiated only by UV and the heating source of the gas at the surface disk is grain photoelectric heating only. If the disk is irradiated by X-rays only, the ratio does not change with the maximum dust size. We note, however, that we have neglected the change in the X-ray photoionization cross section due to the dust evolution. If we take it into account, it affects the line ratio slightly, with the error being about $25 \%$ for the model with $a_{\max }=10 \mathrm{~cm}$ in the extreme case that the contribution to the cross sections of heavy elements in the dust is negligible (see $\S 3.2 .2$; Wilms et al. 2000). Figure 17 shows that the line ratio in the model with X-ray irradiation only is slightly larger than that in the model with UV irradiation, although the gas temperature is lower. This occurs because the level populations are not in LTE, but affected by the X-ray pumping process (see $\S 3.2 .1$ ). We also plot in the right panel of Figure 17 the resulting line ratios for dust model $\mathrm{B}$ in quiescent and turbulent disks as filled and open triangles, respectively. The ratio for the model with dust grains typical of dense clouds (model A with $a_{\max }=$ $10 \mu \mathrm{m}$ ) is plotted as a filled circle. The results suggest that if the dust grains coagulate and settle toward the disk midplane, the ratio becomes substantially larger than the case in which dust grains do not evolve. So, our results suggest that dust evolution in protoplanetary disks could be observable through this particular line ratio. Itoh et al. (2003) derived an upper limit to the line ratio of 0.26 from an observation toward a CTTS, $\mathrm{LkH} \alpha 264$, and J. S. Bary et al. (2007, in preparation) find an upper limit of $\sim 0.2$ toward a Herbig Be star, HD 97048; all the models we used in this paper almost satisfy these observational upper limits.

\section{SUMMARY}

We have made a detailed model of the physical structure of protoplanetary disks and calculated the level populations and line emission of molecular hydrogen, taking into account X-ray irradiation 
from the central star, as well as dust growth and settling toward the disk midplane.

We have followed the time evolution of the spatial and size distributions of dust particles in the disks by numerically solving the coagulation equation for settling dust particles. The resulting mass and total surface area of dust grains per unit gas volume are much smaller, except at the disk midplane, than those for the model in which the dense cloud dust grains are well mixed with the gas. At the disk surface the dust density normalized by the initial value, $\rho_{\text {dust }} / \rho_{\text {dust }, 0}$, and the parameter for the total surface area of dust grains, $f_{\text {dust }}$, are small due to the dust settling toward the disk midplane. Near the disk midplane the parameter $f_{\text {dust }}$ becomes much smaller since small dust particles are removed by dust coagulation.

We have studied the effects of X-ray irradiation on the physical structure of the disks and found that the X-ray irradiation is the dominant heating source in the inner region and in the surface layers of the disk. FUV heating dominates in the middle layers and in the outer region of the disk. This is because the FUV radiation is scattered efficiently by dust grains, while the Compton scattering of X-ray radiation is inefficient in the energy range of $E \lesssim 1 \mathrm{keV}$ in which T Tauri stars mainly emit X-ray radiation. We found that the ionization rate caused by X-rays is much smaller than that due to interstellar cosmic rays near the disk midplane because of the relatively large attenuation and inefficient scattering of X-rays.

The dust evolution in the disks affects the physical disk structure, especially the gas temperature at the disk surface and the FUV radiation field within the disk. As the dust particles grow or settle toward the disk midplane, the gas temperature in the middle layers and the outer disk decreases because the grain photoelectric heating that is induced by FUV radiation becomes less efficient. Meanwhile, the FUV radiation from the central star penetrates deeper into the disk due to the decrease of grain opacity.

Furthermore, making use of the obtained physical structure of the disks, we calculated the level populations of molecular hydrogen in the ground electronic state. Our results show that if the central star has strong X-ray and weak UV radiation, the level populations are controlled by X-ray pumping. Otherwise, the level populations are mainly controlled by thermal collisions or UV pumping, depending on the dust properties in the disk. As the dust particles evolve in the disk, the level populations change from LTE to non-LTE distributions since collisional excitation becomes less efficient due to the decrease of the gas temperature at the disk surface while UV pumping becomes more efficient owing to the stronger UV radiation field in the disk.

Finally, using these level populations, we calculated the line emission of molecular hydrogen from the disk. The rovibrational line spectra in the NIR wavelength band show that the emission lines from high-energy levels become relatively stronger as the dust particles evolve in the disk. Again, this is due to level populations changing from LTE to non-LTE and the populations in higher vibrational energy levels becoming relatively larger. For the pure rotational line spectra in the MIR wavelength band, it is the emission lines from lower energy levels that become relatively stronger. This is because the level populations in the ground vibrational state are in LTE and the populations in lower energy levels become relatively larger with dust evolution and decreasing dust surface area, which results in lower gas temperatures. For transitions in the UV wavelength band, the dependence on the dust evolution is not so straightforward because the line fluxes decrease as the area of the high-temperature region shrinks, while they increase as UV irradiation from the central star penetrates deeper into the disks. Basically, the line fluxes that originate from pumping from higher energy levels seem to be relatively weaker as the dust particles evolve and the gas temperature decreases. Our results suggest that infrared line ratios of molecular hydrogen could be a useful probe of dust evolution in protoplanetary disks. If the dust particles evolve, the $v=1 \rightarrow 0 S(1) / v=2 \rightarrow 1 S(1)$ line ratio, for example, becomes clearly larger than that for the dense cloud dust model (without the dust evolution). Further observations of the line ratios of molecular hydrogen could provide some constraints on the dust evolution model in protoplanetary disks.

We are grateful to an anonymous referee for comments that improved the clarity of our discussion. We would like to thank J. S. Bary, D. A. Weintraub, M. J. Richter, and M. A. Bitner for giving us information about unpublished observational data, Y. Itoh and T. Takeuchi for fruitful comments, and T. Matsuda for useful help with numerical calculations. This work is supported by "The 21 st Century COE Program of Origin and Evolution of Planetary Systems" and Grants-in-Aid for Scientific Research 17039008, 17540217, and 18026006 from MEXT. Astrophysics at Queen's University Belfast is supported by PPARC. H. N. and M. T. acknowledge financial support from the Japan Society for the Promotion of Science.
Adachi, I., Hayashi, C., \& Nakazawa, K. 1976, Prog. Theor. Phys., 56, 1756

Aikawa, Y., \& Herbst, E. 1999, A\&A, 351, 233

2001, A\&A, 371, 1107

Aikawa, Y., \& Nomura, H. 2006, ApJ, 642, 1152

Ardila, D. R., Basri, G., Walter, F. M., Valenti, J. A., \& Johns-Krull, C. M. 2002, ApJ, 566, 1100

Bary, J. S., Weintraub, D. A., \& Kastner, J. H. 2002, ApJ, 576, L73 2003, ApJ, 586, 1136

Beckwith, S., Gatley, I., \& Persson, S. E. 1978, ApJ, 219, L33

Beckwith, S. V. W., \& Sargent, A. I. 1993, in Protostars and Planets III, ed. E. H. Levy \& J. Lunine (Tuscon: Univ. Arizona Press), 521

Bergin, E. A., Aikawa, Y., Blake, G. A., \& van Dishoeck, E. F. 2007, in Protostars and Planets V, ed. B. Reipurth, D. Jewitt, \& K. Keil (Tuscon: Univ. Arizona Press), 751

Bergin, E. A., Calvet, N., D’Alessio, P., \& Herczeg, G. J. 2003, ApJ, 591, L159

Bergin, E. A., et al. 2004, ApJ, 614, L133

Bitner, M. A., Richter, M. J., Lacy, J. H., Greathouse, T. K., Jaffe, D. T., \& Blake, G. A. 2007, ApJ, submitted

Black, J. H., \& van Dishoeck, E. F. 1987, ApJ, 322, 412

Bohren, C. F., \& Huffman, D. R. 1983, Absorption and Scattering of Light by Small Particles (New York: Wiley)
Brown, A., Millar, T. J., Williams, P. M., \& Zealey, W. J. 1983, MNRAS, 203, 785

Burton, M. G., Bulmer, M., Moorhouse, A., Gaballe, T. R., \& Brand, P. W. J. L. 1992, MNRAS, 257, 1P

Calvet, N., D’Alessio, P., Hartmann, L., Wilner, D., Walsh, A., \& Sitko, M. 2002, ApJ, 568, 1008

Carr, J. S. 1989, ApJ, 345, 522

Cazaux, S., \& Tielens, A. G. G. M. 2002, ApJ, 575, L29

. 2004, ApJ, 604, 222

Costa, V. M., Lago, M. T. V. T., Norci, L., \& Meurs, E. J. A. 2000, A\&A, 354, 621

Cuzzi, J. N., Dobrovolskis, A. R., \& Champney, J. M. 1993, Icarus, 106, 102 D’Alessio, P., Calvet, N., \& Hartmann, L. 2001, ApJ, 553, 321

D’Alessio, P., Calvet, N., Hartmann, L., Franco-Hernandez, R., \& Servin, H. 2006, ApJ, 638, 314

D’Alessio, P., Calvet, N., Hartmann, L., Lizano, S., \& Canto, J. 1999, ApJ, 527, 893

Duchene, G., McCabe, C., Ghez, A. M., \& Macintosh, B. A. 2004, ApJ, 606, 969

Dullemond, C. P., \& Dominik, C. 2004, A\&A, 421, 1075

- 2005, A\&A, 434, 971 
Dullemond, C. P., Hollenbach, D., Kamp, I., \& D’Alessio, P. 2007, in Protostars and Planets V, ed. B. Reipurth, D. Jewitt, \& K. Keil (Tuscon: Univ. Arizona Press), 555

Dullemond, C. P., \& Turolla, R. 2000, A\&A, 360, 1187

Dutrey, A., Guilloteau, S., \& Guelin, M. 1997, A\&A, 317, L55

Dutrey, A., Guilloteau, S., \& Ho, P. 2007, in Protostars and Planets V, ed. B. Reipurth, D. Jewitt, \& K. Keil (Tuscon: Univ. Arizona Press), 495

Feigelson, E. D., \& Montmerle, T. 1999, ARA\&A, 37, 363

Getman, K. V., et al. 2005, ApJS, 160, 319

Glassgold, A. E., \& Najita, J. R. 2001, in ASP Conf. Ser. 244, Young Stars near Earth: Progress and Prospects, ed. R. Jayawardhana \& T. P. Greene (San Francisco: ASP), 251

Glassgold, A. E., Najita, J., \& Igea, J. 1997, ApJ, 480, 344

2004, ApJ, 615, 972

Gorti, U., \& Hollenbach, D. 2004, ApJ, 613, 424

Gredel, R., \& Dalgarno, A. 1995, ApJ, 446, 852

Gredel, R., Lepp, S., Dalgarno, A., \& Herbst, E. 1989, ApJ, 347, 289

Hasegawa, T., Gatley, I., Garden, R. P., Brand, P. W. J. L., Ohishi, M., Hayashi, M., \& Kaifu, N. 1987, ApJ, 318, L77

Herbig, G. H., \& Goodrich, R. W. 1986, ApJ, 309, 294

Herbst, W., Herbst, D. K., Grossman, E. J., \& Weinstein, D. 1994, AJ, 108, 1906

Herczeg, G. J., Linsky, J. L., Valenti, J. A., Johns-Krull, C. M., \& Wood, B. E. 2002, ApJ, 572, 310

Herczeg, G. J., Linsky, J. L., Walter, F. M., Gahm, G. F., \& Johns-Krull, C. M. 2006, ApJS, 165, 256

Herczeg, G. J., Wood, B. E., Linsky, J. L., Valenti, J. A., \& Johns-Krull, C. M. 2004, ApJ, 607, 369

Hollenbach, D., \& McKee, C. F. 1989, ApJ, 342, 306

Igea, J., \& Glassgold, A. E. 1999, ApJ, 518, 848

Imanishi, K., Nakajima, H., Tsujimoto, M., Koyama, K., \& Tsuboi, Y. 2003, PASJ, 55, 653

Itoh, Y., Sugitani, K., Ogura, K., \& Tamura, M. 2003, PASJ, 55, L77

Itoh, Y., et al. 2002, PASJ, 54, 963

Jonkheid, B., Dullemond, C. P., Hogerheijde, M. R., \& van Dishoeck, E. F. 2007, A\&A, 463, 203

Jonkheid, B., Faas, F. G. A., van Zadelhoff, G.-J., \& van Dishoeck, E. F. 2004, A\&A, 428, 511

Jonkheid, B., Kamp, I., Augereau, J.-C., \& van Dishoeck, E. F. 2006, A\&A, 453,163

Kaastra, J. S. 1992, An X-Ray Spectral Code for Optically Thin Plasmas (Internal SRON-Leiden Rep., Updated Ver. 2.0)

Kamp, I., \& Dullemond, C. P. 2004, ApJ, 615, 991

Kamp, I., Dullemond, C. P., Hogerheijde, M., \& Enriquez, J. E. 2005, in IAU Symp. 231, Astrochemistry: Recent Successes and Current Challenges, ed. D. C. Lis, G. A. Blake, \& E. Herbst (Cambridge: Cambridge Univ. Press), 377

Kastner, J. H., Huenemoerder, D. P., Schulz, N. S., \& Canizares, C. R. 2002, ApJ, 567, 434

Kenyon, S. J., \& Hartmann, L. 1987, ApJ, 323, 714 1995, ApJS, 101, 117

Kitamura, Y., Momose, M., Yokogawa, S., Kawabe, R., Tamura, M., \& Ida, S. 2002, ApJ, 581, 357

Koyama, K., Maeda, Y., Ozaki, M., Ueno, S., Kamata, Y., Tawara, Y., Skinner, S., \& Yamauchi, S. 1994, PASJ, 46, L125

Kozasa, T., Blum, J., \& Mukai, T. 1992, A\&A, 263, 423

Landau, L. D., Akhiezer, A. I., \& Lifshitz, E. M. 1967, General Physics (Oxford: Pergamon)

Liedahl, D. A., Osterheld, A. L., \& Goldstein, W. H. 1995, ApJ, 438, L115

Maloney, P. R., Hollenbach, D. J., \& Tielens, A. G. G. M. 1996, ApJ, 466, 561

Markwick, A. J., Ilgner, M., Millar, T. J., \& Henning, T. 2002, A\&A, 385, 632

McMaster, W. H., Kerr Del Grande, N., Mallett, J. H., \& Hubbell, J. H. 1969, in Compilation of X-Ray Cross Sections, Lawrence Livermore National Laboratory Rep. UCRL-50174, Sec II, Rev. 1
Mewe, R., Gronenschild, E. H. B. M., \& van den Oord, G. H. J. 1985, A\&AS, 62, 197

Miyake, K., \& Nakagawa, Y. 1993, Icarus, 106, 20

. 1995, ApJ, 441, 361

Mizuno, H. 1989, Icarus, 80, 189

Mizuno, H., Markiewicz, W. J., \& Völk, H. J. 1988, A\&A, 195, 183

Najita, J. R., Carr, J. S., Glassgold, A. E., \& Valenti, J. A. 2007, in Protostars and Planets V, ed. B. Reipurth, D. Jewitt, \& K. Keil (Tuscon: Univ. Arizona Press), 507

Nakagawa, Y., Nakazawa, K., \& Hayashi, C. 1981, Icarus, 45, 517

Nakagawa, Y., Sekiya, M., \& Hayashi, C. 1986, Icarus, 67, 375

Nomura, H. 2002, ApJ, 567, 587

Nomura, H., \& Millar, T. J. 2005, A\&A, 438, 923 (Paper I)

Nomura, H., \& Nakagawa, Y. 2006, ApJ, 640, 1099

Ormel, C. W., Spaans, M., \& Tielens, A. G. G. M. 2007, A\&A, 461, 215

Ossenkopf, V. 1993, A\&A, 280, 617

Pirronello, V., Liu, C., Roser, J. E., \& Vidali, G. 1999, A\&A, 344, 681

Preibisch, T., et al. 2005, ApJS, 160, 401

Qi, C., Wilner, D. J., Calvet, N., Bourke, T. L., Blake, G. A., Hogerheijde, M. R., Ho, P. T. P., \& Bergin, E. 2006, ApJ, 636, L157

Rettig, T., Brittain, S., Simon, T., Gibb, E., Balsara, D. S., Tilley, D. A., \& Kulesa, C. 2006, ApJ, 646, 342

Richter, M. J., Jaffe, D. T., Blake, G. A., \& Lacy, J. H. 2002, ApJ, 572, L161

Richter, M. J., Lacy, J. H., Jaffe, D. T., Greathouse, T. K., \& Blake, G. A. 2007, ApJ, submitted

Roddier, C., Roddier, F., Northcott, M. J., Graves, J. E., \& Jim, K. 1996, ApJ, 463, 326

Sako, S., Yamashita, T., Kataza, H., Miyata, T., Okamoto, Y. K., Honda, M., Fujiyoshi, T., \& Onaka, T. 2005, ApJ, 620, 347

Schmitt, W., Henning, T., \& Mucha, R. 1997, A\&A, 325, 569

Shemansky, D. E., Hall, D. T., \& Ajello, J. M. 1985, ApJ, 296, 765

Sheret, I., Ramsay Howat, S. K., \& Dent, W. R. F. 2003, MNRAS, 343, L65

Stelzer, B., \& Schmitt, J. H. M. M. 2004, A\&A, 418, 687

Sternberg, A., \& Dalgarno, A. 1989, ApJ, 338, 197

Storey, P. J., \& Hummer, D. G. 1995, MNRAS, 272, 41

Suttner, G., \& Yorke, H. W. 2001, ApJ, 551, 461

Takahashi, J., \& Uehara, H. 2001, ApJ, 561, 843

Takeuchi, T., \& Lin, D. N. C. 2005, ApJ, 623, 482

Tanaka, H., Himeno, Y., \& Ida, S. 2005, ApJ, 625, 414

Tanaka, M., Hasegawa, T., Hayashi, S. S., Brand, P. W. J. L., \& Gatley, I. 1989, ApJ, 336, 207

Thi, W., van Dishoeck, E. F., Blake, G. A., van Zadelhoff, G., \& Hogerheijde, M. R. 1999, ApJ, 521, L63

Thi, W.-F., van Zadelhoff, G.-J., \& van Dishoeck, E. F. 2004, A\&A, 425, 955

Thi, W. F., et al. 2001a, ApJ, 561, 1074 2001b, Nature, 409, 60

Tiné, S., Lepp, S., Gredel, R., \& Dalgarno, A. 1997, ApJ, 481, 282

Tsujimoto, M., Koyama, K., Tsuboi, Y., Goto, M., \& Kobayashi, N. 2002, ApJ, 566,974

Umebayashi, T., \& Nakano, T. 1981, PASJ, 33, 617

Valenti, J. A., Johns-Krull, C. M., \& Linsky, J. L. 2000, ApJS, 129, 399

Verner, D. A., \& Yakovlev, D. G. 1995, A\&AS, 109, 125

Wagenblast, R., \& Hartquist, T. W. 1988, MNRAS, 230, 363

Weidenschilling, S. J. 1977, MNRAS, 180, 57 1980, Icarus, 44, 172

. 1997, Icarus, 127, 290
Weingartner, J. C., \& Draine, B. T. 2001, ApJ, 548, 296

Weintraub, D. A., Bary, J. S., Kastner, J. H., Shukla, S. J., \& Chynoweth, K. 2005, in Protostars and Planets V, ed. V. Mannings et al. (Houson: LPI), 8197

Weintraub, D. A., Kastner, J. H., \& Bary, J. S. 2000, ApJ, 541, 767

Wilms, J., Allen, A., \& McCray, R. 2000, ApJ, 542, 914

Zecho, T., Gürtler, A., Sha, X., Jackson, B., \& Küppers, J. 2002, J. Chem. Phys., 117, 8486 